TRANSACTIONS OF THE

AMERICAN MATHEMATICAL SOCIETY

Volume 353, Number 2, Pages 769-794

S 0002-9947(00)02678-7

Article electronically published on October 13, 2000

\title{
ISOMORPHISM PROBLEMS AND GROUPS OF AUTOMORPHISMS FOR GENERALIZED WEYL ALGEBRAS
}

\author{
V. V. BAVULA AND D. A. JORDAN
}

\begin{abstract}
We present solutions to isomorphism problems for various generalized Weyl algebras, including deformations of type-A Kleinian singularities and the algebras similar to $U\left(\mathfrak{s l}_{2}\right)$ introduced by S. P. Smith. For the former, we generalize results of Dixmier on the first Weyl algebra and the minimal primitive factors of $U\left(\mathfrak{s l}_{2}\right)$ by finding sets of generators for the group of automorphisms.
\end{abstract}

\section{INTRODUCTION}

Let $k$ be an algebraically closed field of characteristic 0 and consider the Weyl algebra $A_{1}(k)=k\langle\partial, x: \partial x-x \partial=1\rangle$. Dixmier [1] showed that the $k$-automorphism group of $A_{1}(k)$ is generated by the automorphisms $e^{\lambda \text { ad } x^{n}}$ and $e^{\lambda \text { ad } \partial^{n}}$ where $n \geq 1$ and $\lambda \in k$. Adapting his methods in [12, he found an analogous set of generators for the $k$-automorphism group of the infinite-dimensional primitive factor $B_{\lambda}:=U\left(\mathfrak{s l}_{2}\right) /(C-\lambda)$ of the universal enveloping algebra of the Lie algebra $\mathfrak{s l}_{2}$, where $C$ is the Casimir element and $\lambda \in k$. He also showed that $B_{\lambda} \simeq B_{\lambda^{\prime}}$ if and only if $\lambda=\lambda^{\prime}$.

Let $a \in k[h]$ and let $A(a)$ be the generalized Weyl algebra $k[h](\sigma, a)$, where $\sigma$ is the $k$-automorphism of $k[h]$ such that $\sigma(h)=h-1$. Thus $A(a)$ is the $k$-algebra generated by $h, x$ and $y$ subject to the relations

$$
x h=(h-1) x, y h=(h+1) y, x y=a(h-1), y x=a(h) .
$$

Both $A_{1}(k)$ and $B_{\lambda}$ are of the form $A(a)$ with $\operatorname{deg}(a)=1$ and 2 respectively. Rings of the form $A(a)$, with $a$ of arbitrary degree, were studied by the first author [3] and, under the name deformations of type-A Kleinian singularities, by Hodges [14. The problem of when $A\left(a_{1}\right) \simeq A\left(a_{2}\right)$ was raised in [14, $\left.\S 5(1)\right]$.

Smith 31] gave a substantial analysis of a class of algebras, similar to $U\left(\mathfrak{s i}_{2}\right)$, which can be interpreted as generalized Weyl algebras and which are closely related to the algebras $A(a)$. For $f \in K[H]$, let $R(f)$ denote the $k$-algebra generated by $A, B$ and $H$ subject to the relations

$$
[H, A]=A,[H, B]=-B,[A, B]=f(H) .
$$

The isomorphism problem for these algebras was raised in [31, Remark (2)].

Received by the editors July 12, 1999.

2000 Mathematics Subject Classification. Primary 16S36, 16W20, 16W35.

Key words and phrases. Isomorphism, generalized Weyl algebra, skew polynomial ring, automorphism group, Kleinian singularity.

This work was done during visits to the University of Sheffield by the first author with the support of the London Mathematical Society.

(C)2000 American Mathematical Society 
We shall adapt the methods of Dixmier to find a set of generators for the automorphism group of $A(a)$ and to solve the isomorphism problems for $A(a)$ and $R(f)$. Loosely speaking, we shall see that $A\left(a_{1}\right) \simeq A\left(a_{2}\right)$ if and only if $a_{2}$ can be obtained from $a_{1}$ by a combination of non-zero scalar multiplication, translation $a(h) \mapsto a(h+\tau)$ and a "flip" $a(h) \mapsto a(-h)$ corresponding to an interchange of the roles of $x$ and $y$. The situation for $R(f)$ is analogous. In some cases, including those of $A_{1}(k)$ and $B_{\lambda}$, the flip can be expressed in terms of translation and scalar multiplication and there is an automorphism $\Omega$, well-known for both $A_{1}(k)$ and $B_{\lambda}$, interchanging the roles of $x$ and $y$. We shall say that $a(h)$ is reflective if there exists $\rho \in k$ such that $a(\rho-h)=(-1)^{d} a(h)$, where $d=\operatorname{deg} a$. Polynomials of degree 1 and 2 must be reflective but cubics need not. When $a(h)$ is reflective, there is a $k$-automorphism $\Omega$ of $A(a)$ such that $\Omega(x)=y, \Omega(y)=(-1)^{d} x$ and $\Omega(h)=1+\rho-h$. In general, there is an isomorphism $\Lambda: A(a(h)) \rightarrow A(a(-h))$ such that $\Lambda(x)=y, \Lambda(y)=x$ and $\Lambda(h)=1-h$.

Let $G$ denote the subgroup of the $k$-automorphism group Aut Au $A(a)$ generated by the $k$-automorphisms $e^{\lambda \text { ad } x^{m}}$ and $e^{\lambda \text { ad } y^{m}}$, where $\lambda \in k$ and $m \geq 1$, and the $k$-automorphisms $\Theta_{\mu}$ such that $\Theta_{\mu}(x)=\mu x, \Theta_{\mu}(y)=\mu^{-1} y$ and $\Theta_{\mu}(h)=h$, where $\mu \in k^{*}$. We shall prove:

- (Theorem 3.29) If $a$ is reflective, then $\operatorname{Aut}_{k} A(a)$ is generated by $G$ and $\Omega$. If $a$ is not reflective, then $\operatorname{Aut}_{k} A(a)=G$.

- (Theorem 3.28) For $a_{1}, a_{2} \in k[h], A\left(a_{1}\right) \simeq A\left(a_{2}\right)$ if and only if $a_{2}(h)=$ $\eta a_{1}(\tau \pm h)$ for some $\eta, \tau \in k$ with $\eta \neq 0$.

- (Theorem 4.2) For $f_{1}, f_{2} \in k[H], R\left(f_{1}\right) \simeq R\left(f_{2}\right)$ if and only if $f_{2}(H)=$ $\eta f_{1}(\tau \pm H)$ for some $\eta, \tau \in k$ with $\eta \neq 0$.

Theorem 4.2 is deduced from Theorem 3.28 using the fact that each of the algebras $R(f)$ has a distinguished central element $C$ such that $R(f) / C R(f) \simeq A(a)$ for some $a$. Notice that when $a_{1}$ is reflective the condition for isomorphism in Theorem 3.28 becomes $a_{2}(h)=\eta a_{1}(h+\tau)$.

We shall also prove, in $\S 5$, an analogue of Theorem 4.2 for the algebras which are similar to the quantized enveloping algebra $U_{q}\left(\mathfrak{s l}_{2}\right)$ in the way that the algebras $R(f)$ are similar to $U\left(\mathfrak{s l}_{2}\right)$.

Another isomorphism problem concerns two deformations of $\mathfrak{s l}_{2}$ considered by Witten [33, (5.2) and (5.12)] and a third deformation due to Woronowicz 34. In $\S 6$, we shall show that Witten's second deformation and Woronowicz's deformation are isomorphic. The background to this includes remarks in the literature of mathematical physics [33, 10, suggesting at least that these two algebras become isomorphic after localization or completion and a comment in 24], where a rigorous algebraic relationship between Witten's two deformations is explained, doubting the existence of a connection with the Woronowicz algebra.

\section{Generalized Weyl algebras and ambiskew polynomial Rings}

2.1. Generalized Weyl algebras. Let $D$ be a ring, let $\sigma$ be an automorphism of $D$ and let $a$ be a central element of $D$. The generalized Weyl algebra (of degree 1) $D(\sigma, a)$, introduced by the first author [3, 4, 5, 7, 8], is the ring extension of $D$ generated by two indeterminates $x, y$ subject to the relations

$$
\begin{gathered}
x d=\sigma(d) x \text { and } y d=\sigma^{-1}(d) y \text { for all } d \in D, \\
x y=\sigma(a), y x=a .
\end{gathered}
$$


These algebras were also studied, without a name, by the second author [18, where $D$ is assumed to be commutative, and, under the name hyperbolic ring, by Rosenberg [28. Generalized Weyl algebras of higher degree were introduced in [4] and studied in [4, 7, 8, 9].

2.2. Ambiskew polynomial rings. The papers [3, 9, 15, 16, 17, 19, 20, 22] are concerned with a class of iterated skew polynomial rings, now called ambiskew polynomial rings [21], which can be viewed as important examples of generalized Weyl algebras.

Let $B$ be a ring, let $\sigma$ be an automorphism of $B$, let $v$ be a central element of $B$ and let $p$ be a central unit in $B$. We denote by $R(B, \sigma, v, p)$ the iterated skew polynomial ring $B[x ; \sigma]\left[y ; \sigma^{-1}, \delta\right]$ where the automorphisms $\sigma^{ \pm 1}$ are extended to $B[x ; \sigma]$ by setting $\sigma^{ \pm 1}(x)=p^{\mp 1} x$ and $\delta$ is the $\sigma^{-1}$-derivation of $B[x ; \sigma]$ such that $\delta(B)=0$ and $\delta(x)=v$. Thus $R(B, \sigma, v, p)$ is the ring extension of $B$ generated by $x$ and $y$ subject to the relations

$$
\begin{gathered}
x b=\sigma(b) x \text { and } y b=\sigma^{-1}(b) y \text { for all } b \in B, \\
x y-p y x=v .
\end{gathered}
$$

2.3. Example. The first Weyl algebra $A_{1}(k)=k\langle\partial, x \mid \partial x-x \partial=1\rangle$ over a field $k$ is a basic example for both constructions. To view $A_{1}(k)$ as a generalized Weyl algebra, one first adjoins $h=y x$ to obtain the polynomial ring $k[h]$. Then $A_{1}(k)=$ $k[h](\sigma, h)$, where $\sigma$ is the $k$-automorphism of $k[h]$ such that $\sigma(h)=h-1$. Thus, in $k[h](\sigma, h), x y=h-1, y x=h$ and $y x-x y=1$. When viewing $A_{1}(k)$ as an ambiskew polynomial ring, the base ring is $k$ and $A_{1}(k)=R(k, \mathrm{id},-1,1)$. This illustrates the relationship between the two constructions.

2.4. Lemma. Every ambiskew polynomial ring $R(B, \sigma, v, p)$ is a generalized Weyl algebra $B[w](\sigma, w)$, where $w=y x$ and $\sigma$ is extended to $B[w]$ by setting $\sigma(w)=$ $p w+v$.

Proof. See [22, 2.6 Corollary] or [8, Lemma 1.2].

Every generalized Weyl algebra occurs as a homomorphic image of an ambiskew polynomial ring, obtained by factoring out a normal element $z$ which exists under a certain condition on $v$. When this holds, the previous lemma can be sharpened by replacing $w$ by $z$, which is an eigenvector for $\sigma$.

2.5. Lemma. Let $R=R(B, \sigma, v, p)$. Suppose that $v$ has the form $\sigma(u)-p u$, for some central element $u$ of $B$, and let $z=y x-u=p^{-1}(x y-\sigma(u))$.

(i) $z$ is normal in $R$, with $x z=p z x, y z=p^{-1} z y$ and $z b=b z$ for all $b \in B$, and $R / z R$ is the generalized Weyl algebra $B(\sigma, u)$.

(ii) $R$ is the generalized Weyl algebra $B[z](\sigma, z+u)$, where $\sigma$ is extended to $B[z]$ by setting $\sigma(z)=p z$.

2.6. $\mathbb{Z}$-grading. Every generalized Weyl algebra $A=D(\sigma, a)$ is $\mathbb{Z}$-graded, $A=$ $\bigoplus_{n \in \mathbb{Z}} A_{n}$, where $A_{n}=D x^{n}$ if $n \geq 0$ and $A_{n}=D y^{-n}$ if $n<0$. It follows from the defining relations that, for each positive integer $n$,

$$
y^{n} x^{n}=a \sigma^{-1}(a) \cdots \sigma^{-(n-1)}(a) \text { and } x^{n} y^{n}=\sigma(a) \sigma^{2}(a) \cdots \sigma^{n}(a) .
$$

The next lemma gives details of some automorphisms and isomorphisms for generalized Weyl algebras. 
2.7. Lemma. Let $A=D(\sigma, a)$ be a generalized Weyl algebra and let $\tau$ be a ring automorphism of $D$.

(i) $A \simeq D\left(\sigma^{-1}, \sigma(a)\right)$ with $x \mapsto y, y \mapsto x$ and $d \mapsto d$ for all $d \in D$.

(ii) If $\lambda$ is a central unit in $D$, then $A \simeq D(\sigma, a \lambda)$, with $x \mapsto x \lambda^{-1}, y \mapsto y$ and $d \mapsto d$ for all $d \in D$. In particular, if $a$ is a unit then $D(\sigma, a) \simeq D\left[x^{ \pm 1} ; \sigma\right]$.

(iii) With $\lambda$ as in (ii), there is an automorphism $\Theta_{\lambda}$ of $A$ with $x \mapsto x \lambda, y \mapsto \lambda^{-1} y$ and $d \mapsto d$ for all $d \in D$.

(iv) The automorphism $\tau$ extends to isomorphisms $\tau^{e+}: A \rightarrow D\left(\tau \sigma \tau^{-1}, \tau(a)\right)$, with $\tau^{e+}(x)=x$ and $\tau^{e+}(y)=y$, and $\tau^{e-}: A \rightarrow D\left(\tau \sigma^{-1} \tau^{-1}, \tau \sigma(a)\right)$, with $\tau^{e-}(x)=y$ and $\tau^{e-}(y)=x$.

(v) If $\tau \sigma=\sigma \tau$, then $\tau^{e+}: A \rightarrow D(\sigma, \tau(a))$.

(vi) If $\tau \sigma=\sigma^{-1} \tau$, then $\tau^{e-}: A \rightarrow D(\sigma, \tau \sigma(a))$.

Proof. The proof is routine. In (iv), $\tau^{\mathrm{e}-}$ is obtained from $\tau^{\mathrm{e}+}$ by composition with the isomorphism $D\left(\tau \sigma \tau^{-1}, \tau(a)\right) \rightarrow D\left(\tau \sigma^{-1} \tau^{-1}, \tau \sigma(a)\right)$ given by (i).

The next lemma gives the details for the corresponding automorphisms and isomorphisms for ambiskew polynomial rings.

2.8. Lemma. Let $R=R(B, \sigma, v, p)$ be an ambiskew polynomial ring and let $\tau$ be a ring automorphism of $B$.

(i) $R \simeq R\left(B, \sigma^{-1},-p^{-1} v, p^{-1}\right)$ with $x \mapsto y, y \mapsto x$ and $b \mapsto b$ for all $b \in B$.

(ii) If $\lambda$ is a central unit in $B$ and $\sigma(\lambda)=\lambda$, then $R \simeq R(B, \sigma, v \lambda, p)$, with $x \mapsto \lambda^{-1} x, y \mapsto y$ and $b \mapsto b$ for all $b \in B$.

(iii) With $\lambda$ as in (ii), there is an automorphism of $R$ with $x \mapsto \lambda x, y \mapsto \lambda^{-1} y$ and $b \mapsto b$ for all $b \in B$.

(iv) There are isomorphisms $\tau^{e+}: R \rightarrow R\left(B, \tau \sigma \tau^{-1}, \tau(v), \tau(p)\right)$ and $\tau^{e-}: R \rightarrow$ $R\left(B, \tau \sigma^{-1} \tau^{-1}, \tau\left(p^{-1} v\right), \tau\left(p^{-1}\right)\right.$, extending $\tau$, such that $\tau^{e+}(x)=x, \tau^{e+}(y)=$ $y, \tau^{e-}(x)=y$ and $\tau^{e-}(y)=-x$.

(v) If $\tau \sigma=\sigma \tau$, then $\tau^{e+}: R \rightarrow R(B, \sigma, \tau(v), \tau(p))$.

(vi) If $\tau \sigma=\sigma^{-1} \tau$, then $\tau^{e-}: R \rightarrow R\left(B, \sigma, \tau\left(p^{-1} v\right), \tau\left(p^{-1}\right)\right)$.

Proof. The proof is again routine, though here the construction of $\tau^{\mathrm{e}-}$ from $\tau^{\mathrm{e}+}$ also involves (ii) with $\lambda=-1$.

Two points to note, in comparing Lemmas 2.7 and 2.8, are the extra condition on $\lambda$ in Lemma 2.8 which will be significant in $\S 5$, and the appearance of $\tau\left(p^{-1} v\right)$ rather than $\tau \sigma(v)$ in 2.8 (vi).

2.9. Notation. In the remainder of the paper, $k$ will denote an algebraically closed field and $k^{*}$ will denote the multiplicative group of $k$.

\section{Deformations of type-A Kleinian singularities}

In this section we concentrate on the case where $D$ is the polynomial ring $k[h]$ and $\sigma(h)=h-1$. We assume throughout the section that char $k=0$. Let $a=$ $a(h) \in k[h]$ and let $A(a)=k[h](\sigma, a)$. Thus $A(a)$ is the $k$-algebra generated by $h, x$ and $y$ subject to the relations

$$
x h=(h-1) x, y h=(h+1) y, x y=a(h-1), y x=a(h) .
$$

Examples include the Weyl algebra $A_{1}(k)$ and, for $\lambda \in k$, the algebra $B_{\lambda}:=$ $U\left(\mathfrak{s l}_{2}\right) /(C-\lambda)$, where $C$ is the Casimir element and $a=-h^{2}-h-\frac{\lambda}{4}$; see 3.2 below. 
A lot is known about the algebras $A(a)$ through studies by the first author [3, 4, 5, 7, 9] and by Hodges [14]. The global dimension of $A(a)$ was computed in 3, 4, 14 generalizing a result of Stafford 32] for $B_{\lambda}$. The Krull dimension of $A(a)$ was determined in [4, 14] and the simple $A(a)$-modules were classified in [4, 7]. In [3, 4], it was shown that the spaces $\mathrm{Ext}^{i}$ and Tor $_{i}$ for simple $A(a)$-modules are finite dimensional, generalizing a result of McConnell and Robson [25] on $\mathrm{Ext}^{i}$ in the case of $A_{1}(k)$. When the defining polynomial $a$ has no multiple root, the Grothendieck group $K_{0}(A(a))$ was computed in [14. The two-sided ideals for certain generalized Weyl algebras more general than $A(a)$ were classified in [5, 6]. In [4] Theorem 7], it was shown that if $a \notin k$ and $f \in A(a) \backslash k$, then the centralizer $C(f)$ is commutative and is a free $k[f]$-module of finite rank and this was applied to determine the structure of commutative subalgebras of $A(a)$, generalizing a result of Amitsur [1] for $A_{1}(k)$.

As observed in [14, Theorem 2.1(iii)], the algebra $A(a)$ has a filtration by finitedimensional subspaces, with $\operatorname{deg} x=\operatorname{deg} y=d$ and $\operatorname{deg} h=2$, such that $\operatorname{gr} A(a)$ is isomorphic to the commutative algebra $k[X, Y, Z] /\left(X Y-Z^{d}\right)$. It follows from [26, 8.2.14(i) and 8.6.5] or [23] Theorem 4.5 and Proposition 6.6] that the GelfandKirillov dimension $\operatorname{GK}(A(a))=2$.

The isomorphism problem for the algebras $A(a)$ was raised in [14, p. 289 (1)]. The solution to this will be given in Theorem 3.28 where it will be shown that the only situations where $A\left(a_{1}\right)$ and $A\left(a_{2}\right)$ are isomorphic arise when $a_{2}$ can be obtained from $a_{1}$ by a sequence of transformations of the three types specified in the following lemma.

3.1. Lemma. (i) For all $\lambda \in k^{*}$, there is an isomorphism $\Gamma_{\lambda}: A(a) \rightarrow A(\lambda a)$ such that

$$
\Gamma_{\lambda}(x)=\lambda^{-1} x, \Gamma_{\lambda}(y)=y \text { and } \Gamma_{\lambda}(h)=h .
$$

(ii) For all $\mu \in k$ there is an isomorphism $\operatorname{tr}_{\mu}: A(a(h)) \rightarrow A(a(h+\mu))$ such that

$$
\operatorname{tr}_{\mu}(x)=x, \operatorname{tr}_{\mu}(y)=y \text { and } \operatorname{tr}_{\mu}(h)=h+\mu .
$$

(iii) There is an isomorphism $\Lambda: A(a(h)) \rightarrow A(a(-h))$ such that

$$
\Lambda(x)=y, \Lambda(y)=x \text { and } \Lambda(h)=1-h .
$$

Proof. Here (i) is a special case of Lemma 2.7(ii) while (ii) and (iii) follow from Lemma 2.7(v, vi) with $\operatorname{tr}_{\mu}=\tau^{\mathrm{e}+}$ and $\Lambda=\eta^{\mathrm{e}-}$, where $\tau(h)=h+\mu$, so that $\tau \sigma=\sigma \tau$, and $\eta(h)=1-h$, so that $\eta \sigma(h)=-h=\sigma^{-1} \eta(h)$. Note that $\Lambda^{-1}: A(-h) \rightarrow A(h)$ is defined by the same formulae as $\Lambda: \Lambda^{-1}(x)=y, \Lambda^{-1}(y)=x$, and $\Lambda^{-1}(h)=$ $1-h$.

3.2. Example ([14, Example 4.7]). Taking $a=-h^{2}-h-\frac{\lambda}{4}$, where $\lambda \in k$, and making the change of variables $x \mapsto e, y \mapsto f, h \mapsto 2 h$, we obtain the infinitedimensional primitive factors $B_{\lambda}$ of $U\left(\mathfrak{s l}_{2}\right)$ [12]. Since an arbitrary polynomial of degree 2 can be written in the form $\mu\left((h+\eta)^{2}+(h+\eta)+\frac{\lambda}{4}\right)$, it follows from Lemma 3.1 that if $\operatorname{deg} a=2$, then $A(a) \simeq B_{\lambda}$ for some $\lambda \in k$. Similar calculations show that if $\operatorname{deg} a=1$, then $A(a) \simeq A_{1}(k)$.

The fixed ring of $A_{1}(k)$ for the action of the cyclic group generated by the automorphism $y \mapsto \omega y, x \mapsto \omega^{-1} x$, where $\omega$ is a primitive $n$th root of unity, has the form $A(a)$ with $\operatorname{deg} a=n$. See [14] Example 4.8] for details. 
The solution of the isomorphism problem for the algebras $B_{\lambda}$ was given by Dixmier [12] and our approach is based on his, which in turn was based on his earlier analysis of the Weyl algebra $A_{1}(k)[11]$.

3.3. Classification of elements. The following definitions and notation are from [11, 6.1] and [12 3.1]. Let $R$ be a $k$-algebra, let $w \in R$ and let $\lambda \in k$. Denote by ad $w$ the inner derivation of $R$ defined by $w$, that is $(\operatorname{ad} w)(z)=w z-z w$. Let $\mathcal{D}(w, \lambda):=\{z \in R:(\operatorname{ad} w)(z)=\lambda z\}$ and let $\mathcal{F}(w, \lambda):=\left\{z:(\operatorname{ad} w-\lambda)^{i}(z)=\right.$ 0 for sufficiently large $i\}$. Let $\mathcal{D}(w):=\bigoplus_{\lambda \in k} \mathcal{D}(w, \lambda)$ and $\mathcal{F}(w):=\bigoplus_{\lambda \in k} \mathcal{F}(w, \lambda)$. The elements $z$ of $\mathcal{F}(w)$ are those for which the subspace $\sum_{n=0}^{\infty} k(\operatorname{ad} w)^{n}(z)$ is finite-dimensional. Let $\mathcal{N}(w)=\mathcal{F}(w, 0)$ and observe that $\mathcal{D}(w, 0)$ is the centralizer $\mathcal{C}(w)$ of $w$ in $R$.

The element $w$ is said to be strictly semisimple if $\mathcal{D}(w)=R$ in other words, if the derivation ad $w$ is diagonalizable. In the generalized Weyl algebra $A(a)$, for each $n \in \mathbb{Z}, \mathcal{D}(h, n)=A_{n}$, the $n$th component of $A(a)$ in the $\mathbb{Z}$-grading of $A(a)$ in 92.6. Thus $h$ is strictly semisimple. Note that $\mathcal{C}(h)=k[h]$ and it follows from the formula $x f(h)=f(h-1) x$ that the centre of $A(a)$ is $k$.

The element $w$ is said to be strictly nilpotent (with respect to the adjoint action) if $\mathcal{N}(w)=A$, in other words, if ad $w$ is locally nilpotent. If $w$ is strictly nilpotent, then there is a well-defined $k$-automorphism $e^{\text {ad } w}$ of $R$ such that, for $r \in R$,

$$
\mathrm{e}^{\operatorname{ad} w}(r)=\sum_{i \geq 0}(\operatorname{ad} w)^{i}(r) / i !
$$

The inverse of $e^{\operatorname{ad} w}$ is $e^{\operatorname{ad}(-w)}$.

We now aim to show that $x$ and $y$ are strictly nilpotent in $A(a)$. For $m \in \mathbb{N}$, let $\Delta_{m}$ denote the linear map $\sigma^{m}-1: k[h] \rightarrow k[h]$, that is $\Delta_{m}(f)=\sigma^{m}(f)-f$ for all $f \in k[h]$. Then $\Delta_{m}$ is a $\sigma^{m}$-derivation of the algebra $k[h]$, that is $\Delta_{m}(f g)=$ $\Delta_{m}(f) g+\sigma^{m}(f) \Delta_{m}(g)$, for all $f, g \in k[h]$. For $i \in \mathbb{N}, \operatorname{ker}\left(\left(\Delta_{m}\right)^{i}\right)=\{f \in k[h]$ : $\operatorname{deg} f<i\}$. Thus $\Delta_{m}$ is locally nilpotent.

Let $f \in k[h]$. For $m \in \mathbb{N}$, direct computations give

$$
\operatorname{ad} x^{m}: x \mapsto 0, f \mapsto \Delta_{m}(f) x^{m}, h \mapsto-m x^{m}, y \mapsto \Delta_{m}(a) x^{m-1} .
$$

For $i \geq 0,\left(\operatorname{ad} x^{m}\right)\left(\Delta_{m}^{i}(a) x^{i m-1}\right)=\Delta_{m}^{(i+1)}(a) x^{(i+1) m-1}$. It follows by induction that, for $i \geq 1$,

$$
\left(\operatorname{ad} x^{m}\right)^{i}(y)=\Delta_{m}^{i}(a) x^{i m-1}
$$

Similarly,

$$
\operatorname{ad} y^{m}: x \mapsto-y^{m-1} \Delta_{m}(a), f \mapsto-y^{m} \Delta_{m}(f), h \mapsto m y^{m}, y \mapsto 0,
$$

and, for $i \geq 1$,

$$
\left(\operatorname{ad} y^{m}\right)^{i}(x)=(-1)^{i} y^{i m-1} \Delta_{m}^{i}(a)
$$

3.4. Lemma. Let $d=\operatorname{deg} a(h)$, let $m \geq 0$ be an integer and let $\lambda \in k$. The inner derivations ad $x^{m}$ and ad $y^{m}$ of $A(a)$ are locally nilpotent. Hence there are $k$-automorphisms $\Psi_{m, \lambda}:=e^{\lambda \text { ad } x^{m}}$ and $\Phi_{m, \lambda}:=e^{\lambda \text { ad } y^{m}}$ of $A(a)$ such that

$$
\Psi_{m, \lambda}: x \mapsto x, h \mapsto h-m \lambda x^{m}, y \mapsto y+\sum_{i=1}^{d} \frac{\lambda^{i}}{i !} \Delta_{m}^{i}(a) x^{i m-1}
$$


and

$$
\Phi_{m, \lambda}: x \mapsto x+\sum_{i=1}^{d} \frac{(-\lambda)^{i}}{i !} y^{i m-1} \Delta_{m}^{i}(a), h \mapsto h+m \lambda y^{m}, y \mapsto y
$$

Proof. As $A(a)$ is generated by $x, y$ and $h$, this is immediate from (6)-(9) and the fact that, for $m \geq 1, \Delta_{m}$ is locally nilpotent.

3.5. Automorphisms. We denote by $G$ the subgroup of $\operatorname{Aut}_{k} A(a)$ generated by the $k$-automorphisms of the following three types:

(i) $\Theta_{\mu}$ for $0 \neq \mu \in k$ (in the notation of Lemma 2.7(iii)),

(ii) $\Psi_{m, \lambda}$ for an integer $m \geq 0$ and $\lambda \in k$,

(iii) $\Phi_{m, \lambda}$ for an integer $m \geq 0$ and $\lambda \in k$.

3.6. Notation. Let $0 \neq a \in k[h]$. For the remainder of this section, we write $A$ for $A(a)$. By Lemma 3.1(i), we can assume that $a$ is monic. We denote by $d$ the degree of $a$, and by $\beta$ the coefficient in $a$ of $h^{d-1}$, thus

$$
a=h^{d}+\beta h^{d-1}+\ldots .
$$

3.7. Remark. When the algebra $B_{\lambda}$ from 3.2 is written in the form $A(a)$ with $a$ monic, $a$ has the form $h^{2}+h+\mu$, so that $a(h-1)=a(-h)$. It follows that, in this case, the isomorphism $\Lambda$ in Lemma 3.1 (iii) is an automorphism. This automorphism is denoted by $\Omega$ in 12 where it is frequently used to justify symmetry of arguments involving $x$ and $y$ ( $e$ and $f$ in [12]). In our more general situation, we can again appeal to symmetry using $\Lambda$. Technical results established for $x$ and $y$ will hold in $A(a(-h))$ and hence for $y$ and $x$ in $A$.

For some choices of $a$, there does exist an automorphism of $A(a)$ generalizing the above automorphism $\Omega$. If there exists $\rho \in k$ such that $a(\rho-h)=(-1)^{d} a(h)$ we shall say that $a$ is reflective. This definition is independent of the generator $h$. In particular, if $t=h+\tau$, where $\tau \in k$, and $a=a(h)=b(t)$, then $a(\rho-h)=(-1)^{d} a(h)$ if and only if $b(\rho+2 \tau-t)=(-1)^{d} b(t)$. Let $\tau=\beta d$ so that the coefficient of $t^{d-1}$ in $a=b(t)$ is 0 . Then $a$ is reflective with $a(\rho-h)=(-1)^{d} a(h)$ if and only if $\rho+2 \tau=0$ and $b(-t)=(-1)^{d} b(t)$. Consequently, if $d$ is even, then $a$ is reflective if and only if $a \in k\left[t^{2}\right]$ and, if $d$ is odd, then $a$ is reflective if and only if $a \in t k\left[t^{2}\right]$. In particular, all quadratics are reflective but a monic cubic $h^{3}+\mu h^{2}+\lambda h+\xi$ is reflective if and only if $27 \xi=9 \mu \lambda-2 \mu^{3}$.

3.8. Lemma. If $a$ is reflective with $a(\rho-h)=(-1)^{d} a(h)$, then there exists an automorphism $\Omega$ of $A(a)$ with $\Omega(x)=y, \Omega(y)=(-1)^{d} x$ and $\Omega(h)=1+\rho-h$.

Proof. In the notation of Lemma 3.1.

$$
\Omega=\Gamma_{(-1)^{d}} \Lambda \operatorname{tr}_{\rho}: A(a) \rightarrow A\left((-1)^{d} a(\rho-h)\right)=A(a) .
$$

3.9. Filtrations. Let $\mathcal{P}(d)$ denote the set of all ordered pairs $(i, j)$ where $i$ and $j$ are non-negative integers such that $i \equiv j \bmod d$. Let $f_{0}(h)=1$ and, for $n \geq 1$, let

$$
f_{n}(h)=h^{n}+\gamma_{n} h^{n-1}, \text { where } \gamma_{n}:=\frac{n(n+2 \beta-d)}{2 d} .
$$

Note that $\left\{f_{n}(h): n \geq 0\right\}$ is a $k$-basis for $k[h]$. It can be easily checked that, for all $i, j \geq 0$,

$$
\gamma_{i+j}=\gamma_{i}+\gamma_{j}+i j / d
$$


If $j>i$, with $j=i+m d$ and $m>0$, then set $U_{(i, j)}=f_{i}(h) x^{m}$ and if $j \leq i$, with $i=j+m d$ and $m \geq 0$, then set $U_{(i, j)}=y^{m} f_{j}(h)$. It follows from 2.6 that $\left\{U_{p}: p \in \mathcal{P}(d)\right\}$ is a $k$-basis for $A$.

Let $\sigma, \rho \in \mathbb{R}$ be such that $\rho+\sigma>0$ and let $H_{\rho, \sigma}$ be the additive submonoid of $\mathbb{R}$ generated by $\sigma$ and $\rho$. For $p=(i, j) \in \mathcal{P}(d)$, let $v_{\rho, \sigma}\left(U_{p}\right)=\rho i+\sigma j \in H_{\rho, \sigma}$. For $h \in H_{\rho, \sigma}$, let $A_{h}$ be the $k$-subspace of $A$ spanned by $\left\{U_{p}: v_{\rho, \sigma}(p) \leq h\right\}$, and let $A_{<h}=\bigcup_{j<h} A_{j}$. We shall see that $\left\{A_{h}\right\}_{h \in H_{\rho, \sigma}}$ is a filtration of $A$ by the ordered monoid $H_{\rho, \sigma}$; that is,

(i) $A=\bigcup_{h \in H_{\rho, \sigma}} A_{h}$,

(ii) $A_{h} \subset A_{k}$ for all $h<k \in H_{\rho, \sigma}$,

(iii) $A_{h} A_{k} \subseteq A_{h+k}$ for all $h, k \in A_{h}$,

(iv) $\bigcap_{h \in H_{\rho, \sigma}} A_{h}=0$.

This coincides with the definition in [29, 1.2.13] except for the reversal of $>$ in (ii). In the language of [27, where only filtrations by $\mathbb{Z}$ are considered, (i) and (iv) would be omitted from the definition of filtration and would say that the filtration is exhaustive and separated. The associated $H_{\rho, \sigma}$-graded ring, gr $A:=$ $\bigoplus_{h \in H_{\rho, \sigma}} A_{h} / A_{<h}$, of $A$ with respect to this filtration can be constructed as for filtrations by $\mathbb{N}$ or $\mathbb{Z},\left[26,27\right.$, 29]. For $r \in A_{h}$ and $s \in A_{k},\left(r+A_{<h}\right)\left(s+A_{<k}\right):=$ $r s+A_{<h+k}$. We shall use the following notation adapted from that used in [26] for filtrations by $\mathbb{N}$ : for $w \in A, \bar{w}:=w+A_{<h}$, where $h=v_{\rho, \sigma}(w):=\min \{h \in H: w \in$ $\left.A_{h}\right\}$. Below we specify a commutative $H_{\rho, \sigma}$-graded algebra which will turn out to be isomorphic to $\operatorname{gr} A$.

3.10. Associated graded rings. Let $\mathcal{P}(d), \sigma, \rho$ and $H_{\rho, \sigma}$ be as in $₫ 3.9$ Let $C^{(d)}$ denote the $k$-subalgebra of the commutative polynomial algebra $k[T, S]$ generated by $T^{d}, S^{d}$ and $T S$. Then $C^{(d)}$ has a $k$-basis consisting of monomials of the form $T^{i} S^{j}$ where $(i, j) \in \mathcal{P}(d)$. For a monomial $T^{i} S^{j} \in C^{(d)}$, let $v_{\rho, \sigma}\left(T^{i} S^{j}\right)=\rho i+\sigma j$ and, for $0 \neq q=\sum \alpha_{i j} T^{i} S^{j} \in C^{(d)}$, let $v_{\rho, \sigma}(q)=\sup _{\alpha_{i j} \neq 0} v_{\rho, \sigma}\left(T^{i} S^{j}\right)$. Let $v_{\rho, \sigma}(0)$ $=-\infty$. Then there is an $H_{\rho, \sigma}$-grading on $C^{(d)}$ such that, for $h \in H_{\rho, \sigma}, C_{h}^{(d)}$ is spanned by those monomials $T^{i} S^{j}$ with $v_{\rho, \sigma}(q)=h$. As indicated above, this will be seen to be the associated $H_{\rho, \sigma}$-graded ring of $A$ for a filtration of $A$ by $H_{\rho, \sigma}$. Furthermore, we shall see that commutation in $A$ induces a Poisson bracket on $C^{(d)}$.

3.11. Poisson bracket. Let $C=C^{(1)}=k[T, S]$. The Poisson bracket on $C$, $\{\}:, C \times C \rightarrow C$, is given by

$$
\{b, c\}=\frac{\partial b}{\partial T} \frac{\partial c}{\partial S}-\frac{\partial b}{\partial S} \frac{\partial c}{\partial T}
$$

Thus

$$
\left\{T^{i} S^{j}, T^{p} S^{q}\right\}=(i q-j p) T^{i+p-1} S^{j+q-1},
$$

from which it follows that, for $d \geq 1$, the subalgebra $C^{(d)}$ is invariant under $\{$, and there is an induced Poisson bracket on $C^{(d)}$. It will be convenient to normalize this bracket and write, for $b, c \in C^{(d)}$,

$$
\{b, c\}_{d}=\frac{1}{d}\left(\frac{\partial b}{\partial T} \frac{\partial c}{\partial S}-\frac{\partial b}{\partial S} \frac{\partial c}{\partial T}\right) .
$$

For $c \in C^{(d)}$, we denote by pad $c$ the Poisson-inner derivation of $C^{(d)}$ given by $(\operatorname{pad} c)(b)=\{c, b\}_{d}$. Thus pad $c=\frac{1}{d}\left(\frac{\partial c}{\partial T} \frac{\partial}{\partial S}-\frac{\partial c}{\partial S} \frac{\partial}{\partial T}\right)$. 
3.12. Lemma. In the notation of [3.9, let $(i, j),(p, q) \in \mathcal{P}(d)$. Then

$$
U_{(i, j)} U_{(p, q)} \equiv U_{(i+p, j+q)}-\frac{j p}{d} U_{(i+p-1, j+q-1)} \bmod A_{\rho(i+p-2)+\sigma(j+q-2)},
$$

and

$$
\left[U_{(i, j)}, U_{(p, q)}\right] \equiv \frac{i q-j p}{d} U_{(i+p-1, j+q-1)} \bmod A_{\rho(i+p-2)+\sigma(j+q-2)} .
$$

Proof. As (15) is an immediate consequence of (14), it suffices to establish (14) and this requires consideration of four cases, two of which split into subcases.

Case 1: $j \leq i$ and $q>p$, with $i=j+d m$ and $q=p+d r$.

Subcase 1A: $r>m$. Here $j+q=(i+p)+d(r-m), U_{(i+p, j+q)}=f_{i+p}(h) x^{r-m}$ and

$$
\begin{aligned}
U_{(i, j)} U_{(p, q)} & =y^{m} f_{j}(h) f_{p}(h) x^{r} \\
& =y^{m} x^{m} f_{j}(h+m) f_{p}(h+m) x^{r-m} \\
& \left.=\left(\prod_{s=0}^{m-1} a(h+s)\right) f_{j}(h+m) f_{p}(h+m) x^{r-m} \quad \text { (by (5) ) }\right) \\
& =g(h) x^{r-m},
\end{aligned}
$$

where $g(h)=\left(\prod_{s=0}^{m-1} a(h+s)\right) f_{j}(h+m) f_{p}(h+m) \in k[h]$ is monic of degree $d m+$ $j+p=i+p$. To establish (14) in this case, it suffices to show that the coefficient of $h^{i+p-1}$ in $g(h)$ is $\gamma_{i+p}-j p / d$. The coefficient of $h^{i+p-1}$ in $g(h)$ is

$$
\begin{aligned}
& \left(\sum_{s=0}^{m-1}(\beta+d s)\right)+\left(j m+\gamma_{j}\right)+\left(p m+\gamma_{p}\right) \\
= & m \beta+d m(m-1) / 2+m(j+p)+\gamma_{j+p}-j p / d \\
= & \gamma_{d m}+d m(j+p) / d+\gamma_{j+p}-j p / d \\
= & \gamma_{d m+j+p}-j p / d \\
= & \gamma_{i+p}-j p / d .
\end{aligned}
$$

Subcase 1B: $r \leq m$. Here $U_{(i+p, j+q)}=y^{m-r} f_{j+q}(h)$ and

$$
\begin{aligned}
U_{(i, j)} U_{(p, q)} & =y^{m} f_{j}(h) f_{p}(h) x^{r} \\
& =y^{m-r} f_{j}(h+r) f_{p}(h+r) y^{r} x^{r} \\
& =y^{m-r}\left(\prod_{s=0}^{r-1} a(h+s)\right) f_{j}(h+r) f_{p}(h+r) \\
& =y^{m-r} g(h),
\end{aligned}
$$

where $g(h)=\left(\prod_{s=0}^{r-1} a(h+s)\right) f_{j}(h+r) f_{p}(h+r) \in k[h]$ is monic of degree $d r+j+p=$ $j+q$. The same calculation as in $1 \mathrm{~A}$, with $m$ replaced by $r$, shows that (14) again holds.

Case 2: $j \leq i$ and $q \leq p$, with $i=j+d m$ and $p=q+d r$. Here $U_{(i+p, j+q)}=$ $y^{m+r} f_{j+q}(h)$ and

$$
\begin{aligned}
U_{(i, j)} U_{(p, q)} & =y^{m} f_{j}(h) y^{r} f_{q}(h) \\
& =y^{m+r} f_{j}(h-r) f_{q}(h) \\
& =y^{m+r} g(h),
\end{aligned}
$$


where $g(h)=f_{j}(h-r) f_{q}(h) \in k[h]$ is monic of degree $j+q$. The coefficient of $h^{j+q-1}$ in $g(h)$ is $-j r+\gamma_{j}+\gamma_{q}=\gamma_{j+q}-(j p) / d$ by (12). Hence (14) holds in this case.

Case 3: $j>i$ and $q>p$, with $j=i+d m$ and $q=p+d r$. Here $U_{(i+p, j+q)}=$ $f_{i+p}(h) x^{m+r}$ and

$$
\begin{aligned}
U_{(i, j)} U_{(p, q)} & =f_{i}(h) x^{m} f_{p}(h) x^{r} \\
& =f_{i}(h) f_{p}(h-r) x^{m+r} \\
& =g(h) x^{m+r},
\end{aligned}
$$

where $g(h)=f_{i}(h) f_{p}(h-r) \in k[h]$ is monic of degree $i+p$. The same calculation as in Case 2, with the interchanges $j \leftrightarrow p$ and $i \leftrightarrow q$, shows that (14) holds again.

Case 4: $j>i$ and $q \leq p$, with $j=i+d m$ and $p=q+d r$.

Subcase 4A: $m>r$. Here $j+q=(i+p)+d(m-r), U_{(i+p, j+q)}=f_{i+p}(h) x^{m-r}$ and

$$
\begin{aligned}
U_{(i, j)} U_{(p, q)} & =f_{i}(h) x^{m} y^{r} f_{q}(h) \\
& =f_{i}(h)\left(\prod_{s=1}^{r} a(h-s-m+r)\right) f_{q}(h-m+r) x^{m-r} \quad \text { (by (5) ) } \\
& =g(h) x^{m-r},
\end{aligned}
$$

where $g(h)=f_{i}(h)\left(\prod_{s=1}^{r} a(h-s-m+r)\right) f_{q}(h-m+r) \in k[h]$ is monic of degree $i+d r+q=i+p$. The coefficient of $h^{i+p-1}$ in $g(h)$ is

$$
\begin{aligned}
& \left(\sum_{s=0}^{r-1}(d(s-m)+\beta)\right)+\gamma_{i}+\gamma_{q}+q(r-m) \\
= & \left.r(-d m+\beta)+d r(r-1) / 2+\gamma_{i+q}-i q / d+q(r-m) \quad \text { (by (12) }\right) \\
= & \gamma_{d r}-r d m+\left(\gamma_{i+q}-i q / d\right)+q(r-m) \\
= & \gamma_{d r+i+q}-d r(i+q) / d-r d m-i q / d+q(r-m) \\
= & \gamma_{i+p}-r j-q j / d \\
= & \gamma_{i+p}-j p / d
\end{aligned}
$$

whence (14) holds.

Subcase 4B: $m \leq r$. Here $i+p=(j+q)+d(r-m), U_{(i+p, j+q)}=y^{m-r} f_{j+q}(h)$ and

$$
\begin{aligned}
U_{(i, j)} U_{(p, q)} & =f_{i}(h) x^{m} y^{r} f_{q}(h) \\
& =y^{r-m} f_{i}(h-r+m)\left(\prod_{s=1}^{r} a(h-s-r+m)\right) f_{q}(h) \\
& =y^{r-m} g(h),
\end{aligned}
$$

where $g(h)=f_{i}(h-r+m)\left(\prod_{s=1}^{r} a(h-s-r+m)\right) f_{q}(h) \in k[h]$ is monic of degree $i+d r+q=i+p$. The same calculation as in $4 \mathrm{~A}$, with the interchanges $m \leftrightarrow r$, $i \leftrightarrow q$ and $j \leftrightarrow p$, then shows that (14) holds again.

3.13. Remark. The proof of Lemma 3.12 shows that

$$
U_{(i, j)} U_{(p, q)}=U_{(i+p, j+q)}-\frac{j p}{d} U_{(i+p-1, j+q-1)}+\sum_{k \geq 2} \lambda_{k} U_{(i+p-k, j+q-k)}
$$

for some scalars $\lambda_{k}=\lambda_{k}((i, j),(p, q))$. 
3.14. Theorem. In the notation of [3.9]

(i) $\left\{A_{h}\right\}_{h \in H_{\rho, \sigma}}$ is a filtration of $A$ by $H_{\rho, \sigma}$.

(ii) For this filtration, gr $A$ is isomorphic to $C^{(d)}$, with the $H_{\rho, \sigma}$-grading on $C^{(d)}$ specified in 3.10. An isomorphism, which we shall use to identify gr $A$ with $C^{(d)}$, is given by

$$
\sum_{(i, j) \in \mathcal{P}(d)} \alpha_{(i, j)} \overline{U_{(i, j)}} \leftrightarrow \sum_{(i, j) \in \mathcal{P}(d)} \alpha_{(i, j)} T^{i} S^{j}
$$

(iii) Let $w_{1}, w_{2} \in A$ and let $z=\left[w_{1}, w_{2}\right]$. For $i=1,2$, let $v_{i}=v_{\rho, \sigma}\left(w_{i}\right)$ and let $q_{i}=\overline{w_{i}}$. Then $v_{\rho, \sigma}\left(w_{1} w_{2}\right)=v_{1}+v_{2}$ and $v_{\rho, \sigma}(z) \leq v_{1}+v_{2}-(\rho+\sigma)$. Moreover, $v_{\rho, \sigma}(z)=v_{1}+v_{2}-(\rho+\sigma) \Leftrightarrow\left\{q_{1}, q_{2}\right\}_{d} \neq 0$, in which case $\bar{z}=\left\{q_{1}, q_{2}\right\}_{d}$.

Proof. This all follows directly from Lemma 3.12 and the fact that $\left\{U_{p}: p \in \mathcal{P}(d)\right\}$ is a $k$-basis for $A$.

The next few results, culminating in Lemma 3.19 are aimed at extending key results, 11. Proposition 7.4] and [12, Proposition 3.3], for the cases $d=1,2$ to higher degree and in particular to the case $d=4$ which turns out to require special attention.

3.15. Lemma. Let $c=\lambda T^{d}+\mu S^{d} \in C^{(d)}$, where $\lambda, \mu \in k^{*}$, and let $n$ be a positive integer. Let $b \in C^{(d)}$. If $\left\{c^{n}, b\right\}_{d}=0$, then $b \in k[c]$.

Proof. We use the $H_{1,1}$-grading on $C^{(d)}$. Suppose that $\{c, b\}_{d}=0$ for some $b \in$ $C^{(d)} \backslash k[c]$ and choose such an element $b$ with $f:=v_{1,1}(b)$ minimal. Let $I=\{i \in \mathbb{Z}$ : $0 \leq i \leq f,(f-i, i) \in \mathcal{P}(d)\}$ and let $u$ be the leading term of $b$. Then

$$
u=\sum_{i \in I} \alpha_{i} T^{f-i} S^{i}
$$

where $\alpha_{i} \in k$ for each $i \in I$, and $\{c, u\}_{d}=0$. Thus

$$
0=\sum_{i \in I} \alpha_{i}\left(i \lambda T^{f+d-i-1} S^{i-1}-(f-i) \mu T^{f-i-1} S^{i+d-1}\right) .
$$

Let $i \in I$. Comparing coefficients of $T^{f+d-i-1} S^{i-1}$ in $(16)$, if $i-d \notin I$, then $i \alpha_{i} \lambda=0$ and if $i-d \in I$, then

$$
i \lambda \alpha_{i}=(f+d-i) \mu \alpha_{i-d} .
$$

It follows that if $i$ is minimal such that $i \in I$ and $\alpha_{i} \neq 0$, then $i=0$ and that if $j \neq \equiv \bmod d$, then $\alpha_{j}=0$. Hence $f \equiv 0 \bmod d$ and we can assume that $\alpha_{0}=\lambda^{m}$, where $m=f / d$. By induction on $r$, it now follows from (17) that, for $0 \leq r \leq m$,

$$
\alpha_{r d}=\left(\begin{array}{c}
m \\
r
\end{array}\right) \lambda^{m-r} \mu^{r} .
$$

Thus $u$ is the leading term of $c^{m}$. Then $\left\{b-c^{m}, c\right\}_{d}=0$, contradicting the minimality of $f$. This completes the proof for the case $n=1$.

For the general case, suppose that $\left\{c^{n}, b\right\}_{d}=0$ and let $\delta$ be the derivation pad $b$ of $C^{(d)}$. Then $n c^{n-1} \delta(c)=\delta\left(c^{n}\right)=0$, so, as $C^{(d)}$ is a domain and char $k=0$, $\delta(c)=0$ and, by the case $n=1, b \in k[c]$.

3.16. Lemma. Let $c=\lambda T^{2}+\mu S^{2} \in C^{(2)}$, where $\lambda, \mu \in k^{*}$, and let $n>0$ and $m \geq 0$ be integers. There does not exist $b \in C^{(2)}$ such that $\left\{c^{n}, b\right\}_{2}=\rho c^{m}$ for some non-zero $\rho \in k$. 
Proof. Again we use the $H_{1,1}$-grading on $C^{(2)}$. Suppose that $\left\{c^{n}, b\right\}_{2}=\rho c^{m}$ for some non-zero $\rho \in k$. As $c^{m}$ and $c^{n}$ are homogeneous with $v_{1,1}\left(c^{n}\right)=2 n$ and $v_{1,1}\left(c^{m}\right)=2 m$, we can assume that $b$ is homogeneous with $v_{1,1}(b)=2(m-n)+$ $2 \geq 2$. Thus $m \geq n$. With $\delta=-\operatorname{pad} b, \rho c^{m}=\delta\left(c^{n}\right)=n c^{n-1} \delta(c)$, whence $\frac{\rho}{n} c^{m-n+1}=\delta(c)=\{c, b\}_{2}$ and we may assume that $n=1$. Thus $\{c, b\}_{2}=\rho c^{m}$ for some non-zero $\rho \in k$ and $b$ is homogeneous with $v_{1,1}(b)=2 m$. The ring $C^{(2)}$ has a $\mathbb{Z}_{2}$-grading in which the even part, $C_{+}$, is spanned by the monomials $T^{i} S^{j}$ with $i$ and $j$ both even and the odd part, $C_{-}$is spanned by the monomials $T^{i} S^{j}$ with $i$ and $j$ both odd. Now $c, c^{m} \in C_{+},\left\{c, C_{+}\right\}_{2} \subseteq C_{-}$and $\left\{c, C_{-}\right\}_{2} \subseteq C_{+}$, so we can assume that $b \in C_{-}$, that is $b$ has the form

$$
T S\left(\sum_{i=0}^{m-1} \alpha_{i} T^{2(m-i-1)} S^{2 i}\right), \alpha_{i} \in k .
$$

Then

$$
\begin{aligned}
\{c, b\}_{2}= & \lambda \sum_{i=0}^{m-1} \alpha_{i}(2 i+1) T^{2(m-i)} S^{2 i} \\
& \quad-\mu \sum_{i=0}^{m-1} \alpha_{i}(2(m-i-1)+1) T^{2(m-i-1)} S^{2(i+1)} \\
= & \lambda \alpha_{0} T^{2 m}-\mu \alpha_{m-1} S^{2 m} \\
& \quad+\sum_{i=1}^{m-1}\left(\lambda \alpha_{i}(1+2 i)-\mu \alpha_{i-1}(1+2(m-i)) T^{2(m-i)} S^{2 i}\right.
\end{aligned}
$$

Setting this equal to $\rho c^{m}=\rho \sum_{i=0}^{m}\left(\begin{array}{c}m \\ i\end{array}\right)\left(\lambda T^{2}\right)^{m-i}\left(\mu S^{2}\right)^{i}$, we see that

(i) $\alpha_{0}=\rho \lambda^{m-1}$;

(ii) for $1 \leq i \leq m-1, \lambda \alpha_{i}(1+2 i)=\mu \alpha_{i-1}(1+2(m-i))+\rho\left(\begin{array}{c}m \\ i\end{array}\right) \lambda^{m-i} \mu^{i}$;

(iii) $\alpha_{m-1}=-\rho \mu^{m-1}$.

From (i) and (ii), it follows inductively that, for $0 \leq i \leq m-1$, there exists $q_{i} \in \mathbb{Q}$, with $q_{i}>0$, such that $\alpha_{i}=\rho q_{i} \lambda^{m-i-1} \mu^{i}$. Here $q_{0}=1$ and, for $i>0$,

$$
q_{i}=\frac{1+2(m-i) q_{i-1}+\left(\begin{array}{c}
m \\
i
\end{array}\right)}{1+2 i}>0 .
$$

In particular, $\alpha_{m-1}=\rho q_{m-1} \mu^{m-1}$, where $q_{m-1}>0$, contradicting (iii).

3.17. Lemma. Let $c=\lambda T^{2}+\mu S^{2} \in C^{(2)}$, where $\lambda, \mu \in k^{*}$, and let $n>0$ and $m \geq 0$ be even integers.

(i) For $b \in C^{(4)}$, if $\left\{c^{n}, b\right\}_{4}=0$, then $b \in k\left[c^{2}\right]$.

(ii) There does not exist $b \in C^{(4)}$ such that $\left\{c^{n}, b\right\}_{4}=\rho c^{m}$ for some non-zero $\rho \in k$.

Proof. Note that $C^{(4)} \subset C^{(2)}$ and $\left\{c^{n}, b\right\}_{2}=2\left\{c^{n}, b\right\}_{4}$.

(i) Let $b \in C^{(4)}$ be such that $\left\{c^{n}, b\right\}_{4}=0$. Then $\left\{c^{n}, b\right\}_{2}=0$ and, by Lemma [3.15, $b \in k[c] \cap C^{(4)}=k\left[c^{2}\right]$. 3.16.

(ii) If $\left\{c^{n}, b\right\}_{4}=\rho c^{m}$, then $\left\{c^{n}, b\right\}_{2}=2 \rho c^{m}$, which is impossible by Lemma 
3.18. Lemma. Let $d=4$ and let $u=c^{2 j}$ for some $j \geq 1$, where $c=\lambda T^{2}+\mu S^{2}$ and $\lambda, \mu \in k^{*}$. Let $b \in C^{(4)} \backslash k\left[c^{2}\right]$ be homogeneous in the $H_{1,1}$-grading. Then $(\operatorname{pad} u)^{i}(b) \neq 0$ for all positive integers $i$.

Proof. Suppose not and choose the least positive integer $i$ such that $(\operatorname{pad} u)^{i}(b)=0$. By 3.17(i), $i>1$. Let $b_{1}=(\operatorname{pad} u)^{i-1}(b)$ and $b_{2}=(\operatorname{pad} u)^{i-2}(b)$, which are non-zero and homogeneous. Then $\left\{c^{2 j}, b_{1}\right\}_{4}=0$ so, by 3.17 (i) and homogeneity, $\left\{c^{2 j}, b_{2}\right\}_{4}=$ $b_{1}=\rho c^{m}$ for some even positive integer $m$. By 3.17(ii), this is impossible.

3.19. Lemma. Let $d=4$ and let $c=\lambda T^{2}+\mu S^{2}$, where $\lambda, \mu \in k^{*}$. Let $w \in A$ and let $u=\bar{w} \in \operatorname{gr} A=C^{(4)}$. If $u=c^{n}$ for some even positive integer $n$, then $\mathcal{F}(w) \neq A$.

Proof. We use the $H_{1,1}$-grading on $C^{(4)}$. Let $z \in A$ be such that, in $\operatorname{gr} A, \bar{z} \notin k\left[c^{2}\right]$, let $b=\bar{z}$ and let $v=v_{1,1}(z)$. For $i \geq 0$, let $z_{i}=(\operatorname{ad} w)^{i}(z)$ and $b_{i}=(\operatorname{pad} u)^{i}(b)$. By Lemma 3.18, $b_{i} \neq 0$ for all $i \geq 0$. Hence, by Theorem 3.14(iii), for each $i, b_{i}=\overline{z_{i}}$ and $v_{1,1}\left(z_{i}\right)=v+i(2 n-2) \rightarrow \infty$ as $i \rightarrow \infty$. Thus $z \notin \mathcal{F}(w)$.

3.20. Lemma. Let $\sigma$ and $\rho$ be positive integers. Let $w \in A$, let $v=v_{\rho, \sigma}(w)$ and let $q=q(T, S)=\bar{w} \in \operatorname{gr} A=C^{(d)}$. Suppose that $v>\rho+\sigma$, that $q$ is not a monomial and that $\mathcal{F}(w)=A$. Then one of the following holds:

(i) $\sigma>\rho, \sigma$ is a multiple of $\rho$, and $q=\xi T^{\alpha}\left(T^{\sigma / \rho}+\mu S\right)^{\beta}$ for some $\xi, \mu \in k^{*}$ and some integers $\alpha \geq 0$ and $\beta>0$.

(ii) $\sigma<\rho, \rho$ is a multiple of $\sigma$, and $q=\xi S^{\alpha}\left(S^{\rho / \sigma}+\mu T\right)^{\beta}$ for some $\xi, \mu \in k^{*}$ and some integers $\alpha \geq 0$ and $\beta>0$.

(iii) $d=1, \sigma=\rho$ and

$$
q=\xi(\mu T+\nu S)^{\alpha}\left(\mu^{\prime} T+\nu^{\prime} S\right)^{\beta}
$$

for some $\xi, \mu, \nu, \mu^{\prime}, \nu^{\prime} \in k$, with $\xi$ and at least three of $\mu, \nu, \mu^{\prime}, \nu^{\prime}$ non-zero, and some integers $\alpha, \beta \geq 0$, with $\alpha+\beta>0$.

(iv) $d=2, \sigma=\rho$ and $q$ has the form in (19) with $\alpha+\beta$ even.

Proof. As was observed in $\$ 3.3$ the centre of $A$ is $k$ so $\mathcal{F}(w) \neq \mathcal{C}(w)$. By the proofs of [11, Lemme 7.3 and Proposition 7.4], either (i) holds or (ii) holds or $\rho=\sigma$ and $q$ is as in (19). Note that the non-zero conditions on the parameters are consequences of the fact that $q \neq 0$ and is not a monomial. An element of the form (19) is in $C^{(2)}$ if and only if $\alpha+\beta$ is even. Thus if $d \leq 2$, then (iii) holds or (iv) holds. Therefore we can assume that $d>2$. To show that if $\rho=\sigma$, then either (iii) or (iv) holds, it suffices to show that if $q \in C^{(d)}$ has the form (19), then $d=4, \alpha$ is even and $q=\gamma\left(\lambda T^{2}+\mu S^{2}\right)^{\alpha}$ for some $\gamma, \lambda, \mu \in k$, with $\gamma \neq 0$ and, as $q$ is not a monomial, $\lambda \neq 0 \neq \mu$. Then, by Lemma 3.19, $\mathcal{F}(w) \neq A$.

We can assume that $\xi=1$. If all four of $\mu, \nu, \mu^{\prime}, \nu^{\prime}$ are non-zero, we can assume that $\mu=1=\mu^{\prime}$. In this case the coefficients in $q$ of $T^{\alpha+\beta-1} S$ and $T S^{\alpha+\beta-1}$ are $\alpha \nu+\beta \nu^{\prime}$ and $\alpha \nu^{\alpha-1} \nu^{\prime \beta}+\beta \nu^{\alpha} \nu^{\prime \beta-1}$ respectively, and as $d>2$ and $q \in C^{(d)}$, so that $d$ divides $\alpha+\beta$ but not $\alpha+\beta-2$, these must be 0 . Thus

$$
\alpha \nu+\beta \nu^{\prime}=0=\alpha \nu^{\prime}+\beta \nu .
$$

As $\alpha$ and $\beta$ cannot both be 0 and neither is negative, it follows that $\nu=-\nu^{\prime}$ and $\beta=\alpha$. Thus $q=\left(T^{2}-\nu^{2} S^{2}\right)^{\alpha}$, which has non-zero coefficients of $T^{2 \alpha}$ and $T^{2 \alpha-2} S^{2}$. Hence $d$ divides $2 \alpha$ and $2 \alpha-4$. As $d>2$ it follows that $d=4$ and $\alpha$ is even. If only three of $\mu, \nu, \mu^{\prime}, \nu^{\prime}$ are non-zero, easy calculations show that either 
the coefficients of $T^{\alpha+\beta}$ and $T^{\alpha+\beta-1} S$ are non-zero or the coefficients of $S^{\alpha+\beta}$ and $T S^{\alpha+\beta-1}$ are not zero and hence, in this case, that $q \notin C^{(d)}$ if $d>2$.

3.21. Automorphisms of gr $A$. Let $n$ be a positive integer and, for $\mu \in k$, consider the automorphism $\Phi_{n, \mu}$. By (11), this is a filtered automorphism for the filtration of $A$ by $H_{1, d n-1}$, that is $\Phi_{n, \mu}\left(A_{h}\right)=A_{h}$ for all $h \in H_{1, d n-1}$. Consequently, it induces an automorphism $\overline{\Phi_{n, \mu}}$ of the associated $H_{1, d n-1 \text {-graded ring }}$ $C^{(d)}$. As the leading term (in the usual grading of $\left.k[h]\right)$ of $\frac{1}{i !} \Delta_{n}^{i}(a)$ is $(-n)^{i}\left(\begin{array}{c}d \\ i\end{array}\right)$, it follows from (11) that $\overline{\Phi_{n, \mu}}$ acts as the restriction to $C^{(d)}$ of the automorphism of $k[T, S]$ such that

$$
T \mapsto T, S \mapsto S+n \mu T^{d n-1}
$$

3.22. Lemma. Let $w \in A$ be of the form

$$
\alpha_{0}+\alpha_{1} x+\alpha_{2} x^{2}+\ldots+\alpha_{r} x^{r}+\alpha h
$$

with $0 \neq \alpha \in k$ and each $\alpha_{j} \in k$. There exist $\Phi \in G$ and $\beta, \beta_{0} \in k$ such that $\Phi(w)=\beta_{0}+\beta h$.

Proof. The proof is as for [12, Lemme 5.1]. For an appropriate value of $\mu, \Phi_{r, \mu}(w)=$ $\alpha_{0}+\alpha_{1} x+\alpha_{2} x^{2}+\ldots+\alpha_{r-1} x^{r-1}+\alpha h$ and the result follows by induction.

3.23. Lemma. Let $w=\sum \alpha_{i, j} U_{(i, j)} \in A$. Let $r$ be the least non-negative integer such that $\alpha_{i, 0}=0$ whenever $i>r$. Let $s$ be the least non-negative integer such that $\alpha_{0, j}=0$ whenever $j>s$. Suppose that there exist integers $i_{1}, j_{1}$ such that:

(1) $\alpha_{i_{1}, j_{1}} \neq 0$;

(2) $\left(i_{1}, j_{1}\right) \neq(1,1)$;

(3) either $s i_{1}+r j_{1}>$ rs or $r=s=0$ and $\left(i_{1}, j_{1}\right) \neq(0,0)$. Then $\mathcal{F}(w) \neq A$.

Proof. The proof is an adaptation of the proof of [12, Lemme 5.2], which in turn was adapted from the proof of [11, Lemme 8.7]. As in [12, by hypothesis (3), $i_{1}>0, j_{1}>0$ and there exist positive real numbers $\rho, \sigma$, linearly independent over $\mathbb{Q}$, such that $\sigma i_{1}+\rho j_{1}>\rho s$ and $\sigma i_{1}+\rho j_{1}>\sigma r$. There exist $i_{2}, j_{2} \geq 0$ such that $\alpha_{i_{2}, j_{2}} \neq 0, v_{\rho, \sigma}(w)=\sigma i_{2}+\rho j_{2}$ and, in $\operatorname{gr} A$ for the filtration of $A$ by $H_{\rho, \sigma}$, $\bar{w}=\alpha_{i_{2}, j_{2}} T^{i_{2}} S^{j_{2}}$. As in [12], $i_{2}>1$ or $j_{2}>1$. By symmetry (see Remark [3.7), we can suppose that $i_{2} \leq j_{2}$.

For $n \geq 0$, let $x_{n}=\delta^{n}(x)$, where $\delta=\operatorname{ad} w$. As in [12, one shows by induction on $n$ that $\overline{x_{n}}=\beta_{n} T^{n\left(i_{2}-1\right)} S^{d+n\left(j_{2}-1\right)}$, for some $0 \neq \beta_{n} \in k$. This is true for $n=0$ as $\bar{x}=S^{d}$. Supposing it to be true for some $n \geq 0$ and applying Theorem 3.14(iii) to $w$ and $x_{n}$, one finds that $\overline{x_{n+1}}=\frac{1}{d}\left(d i_{2}+n j_{2}-n i_{2}\right) \alpha_{i_{2}, j_{2}} \beta_{n} T^{(n+1)\left(i_{2}-1\right)} S^{d+(n+1)\left(j_{2}-1\right)}$. It follows that $v_{\rho, \sigma}\left(x_{n}\right)=\sigma n\left(i_{2}-1\right)+\rho\left(d+n\left(j_{2}-1\right)\right) \rightarrow \infty$ as $n \rightarrow \infty$, whence $x \notin \mathcal{F}(w)$ and $\mathcal{F}(w) \neq A$.

3.24. Remark. In the proofs of the next two lemmas, we can assume that $d>2$ for otherwise, as observed in $\$ 3.2$, either $A \simeq B_{\lambda}$ for some $\lambda$, in which case [12, Lemme 5.3 and Lemme 5.4] are applicable, or $A \simeq A_{1}(k)$, in which case we can apply [11. Lemme 8.8, Théorème 9.2 and proof of Lemme 8.4].

3.25. Lemma. Let $w \in A$ be such that $\mathcal{F}(w)=A$. There exists $\Phi \in G$ such that $\Phi(w) \in k[x]$ or $\Phi(w) \in k[y]$ or $\Phi(w)=\lambda x+\eta h+\mu y+\tau$ for some $\lambda, \eta, \mu, \tau \in k$.

Proof. Again this is adapted from the proofs of [11, Lemme 8.8] and [12, Lemme 5.3]. Let $r=r(w)$ and $s=s(w)$ be as in Lemma [3.23 Write $w=\sum \alpha_{i, j} U_{(i, j)}$. 
Applying Lemmas 3.22 and 3.23 the result is true if $r=0$ or $s=0$ so, by symmetry (see Remark [3.7), it suffices to consider the case where $r \geq s \geq d$. Let $n=r+s$. By induction, we can assume that the result is true whenever $r(w)+s(w)<n$. As $r \geq s>2$, we have $r s>r+s$.

We work with the filtration of $A$ by $H_{s, r}$. By Lemma 3.23, if $\alpha_{i, j} \neq 0$, then either $i=j=1$, in which case $v_{s, r}\left(U_{(i, j)}\right)=s+r<r s$, or $v_{s, r}\left(U_{(i, j)}\right)=i s+j r \leq r s$. As $\alpha_{r, 0} \neq 0$ and $\alpha_{0, s} \neq 0, v_{s, r}(w)=r s$, and $\bar{w}$ has the form

$$
q=\beta_{0} T^{r}+\ldots+\beta_{s} S^{s} \text { where } \beta_{0} \neq 0, \beta_{s} \neq 0 .
$$

As $q \in C^{(d)}, r=j d$ and $s=t d$ for some positive integers $j, t$.

By Lemma 3.20, the possibility that $r=s$ only arises when either $d=1$ or $d=2$. Thus we may suppose that $r>s$. By Lemma 3.20 $s \mid r$ and $q=\lambda\left(T^{m}+\mu S\right)^{s}$ for some $\lambda, \mu \in k^{*}$, where $m=\frac{r}{s}$. As $q \in C^{(d)}, d \mid s$ and, as the coefficient of $T^{m} S^{s-1}$ is non-zero, $d \mid s-1-m$. Thus $d \mid m+1$ and there exist positive integers $n, t$ such that $s=d t, m=d n-1$ and $r=(d n-1) s=(d n-1) d t$. Thus $q=\lambda\left(T^{d n-1}+\mu S\right)^{d t}$ has the form

$$
q=\sum_{i=0}^{d t} \beta_{i} T^{(d n-1)(d t-i)} S^{i} .
$$

Let $\nu \in k$. By (20) $), v_{1, d n-1}\left(\overline{\Phi_{n, \nu}}(q)\right) \leq v_{1, d n-1}(q)=d t(d n-1)$ and the coefficients of $S^{d t}$ and $T^{d t(d n-1)}$ in $\overline{\Phi_{n, \nu}}(q)$ are $\beta_{d t}$ and

$$
\sum_{i=0}^{d t} \beta_{i} n^{i} \nu^{i}
$$

respectively. We can choose $\nu$ so that (23) is 0 . Then $r\left(\Phi_{n, \nu}(w)\right)<r(w)$ and $s\left(\Phi_{n, \nu}(w)\right)=s(w)$ so, by induction there exists $\Phi_{1} \in G$ such that $\Phi_{1}\left(\Phi_{n, \nu}(w)\right) \in$ $k[y]$ or $\Phi_{1}\left(\Phi_{n, \nu}(w)\right)=\lambda x+\eta h+\mu y+\tau$ for some $\lambda, \gamma, \mu, \tau \in k$. The result follows on setting $\Phi=\Phi_{1} \Phi_{n, \nu} \in G$.

3.26. Proposition. Let $w \in A$. Then $w$ is strictly semisimple if and only if there exists $\Phi \in G$ such that $\Phi(w)=\gamma h+\tau$, for some $\gamma, \tau \in k$ with $\gamma \neq 0$.

Proof. Suppose that $w$ is strictly semisimple. Then $w$ is not strictly nilpotent so $w \notin k[y]$ and, by Lemma 3.25 there exists an automorphism $\Phi_{1} \in G$ such that $w_{1}:=\Phi_{1}(w)=\lambda x+\gamma h+\mu y+\tau$ for some $\lambda, \gamma, \mu, \tau \in k$. As $d>2, \overline{w_{1}}=\lambda T^{d}+\mu S^{d}$. By Lemma 3.20 with $\rho=\sigma=1$, this must be a monomial so either $\lambda=0$ or $\mu=0$.

Without loss of generality, suppose $\lambda=0$. Thus $\Phi_{1}(w)=\gamma h+\mu y+\tau$ and, as $y$ is strictly nilpotent, $\gamma \neq 0$. Now let $\Phi=\Phi_{1, \rho} \Phi_{1} \in G$, where $\rho=-\gamma^{-1} \mu$. By (10), $\Phi_{1, \rho}(y)=y$ and $\Phi_{1, \rho}(h)=h+\rho y$, so $\Phi(w)=\gamma h+\tau$.

Conversely, if $w=\Phi^{-1}(\gamma h+\tau)$, for some $\gamma \in k^{*}, \tau \in k$ and $\Phi \in G$, then, as $h$ is strictly semisimple, so too is $w$.

3.27. Theorem. Let $x_{1}, y_{1}, h_{1} \in A$. Suppose that $x_{1}, y_{1}$ and $h_{1}$ generate $A$ and satisfy the relations

$$
x_{1} h_{1}=\left(h_{1}-1\right) x_{1}, y_{1} h_{1}=\left(h_{1}+1\right) y_{1}, x_{1} y_{1}=a_{1}\left(h_{1}-1\right), y_{1} x_{1}=a_{1}\left(h_{1}\right)
$$

for some monic $a_{1} \in k[h]$. There exists $\Phi \in G$ such that, for some $\tau \in k$, either $\Phi\left(x_{1}\right)=x, \Phi\left(y_{1}\right)=y, \Phi\left(h_{1}\right)=h+\tau$ and $a_{1}(h+\tau)=a(h)$, or $\Phi\left(x_{1}\right)=y, \Phi\left(y_{1}\right)=$ $(-1)^{d} x, \Phi\left(h_{1}\right)=\tau-h$ and $(-1)^{d} a_{1}(\tau-h-1)=a(h)$. 
Proof. There is a surjection $A\left(a_{1}\right) \rightarrow A(a)$ so, as $\operatorname{GK}\left(A\left(a_{1}\right)\right)=2=\operatorname{GK}(A(a))$, it follows from [23. Proposition 3.15] that $A\left(a_{1}\right) \simeq A(a)$.

As $h_{1}$ is strongly semisimple, by Lemma [3.26 there exists $\Phi \in G$ such that $\Phi\left(h_{1}\right)=\gamma h+\tau$, for some $\gamma, \tau \in k$, with $\gamma \neq 0$. Let $x_{2}=\Phi\left(x_{1}\right)$ and $y_{2}=\Phi\left(y_{1}\right)$. The set of eigenvalues for ad $h_{1}$ is $\mathbb{Z}$ and the set of eigenvalues for $\operatorname{ad}(\gamma h+\tau)$ is $\gamma \mathbb{Z}$ so $\gamma= \pm 1$.

Suppose that $\gamma=1$, that is $\Phi\left(h_{1}\right)=h+\tau$. In this case $\left[h, x_{2}\right]=\Phi\left(\left[h_{1}, x_{1}\right]\right)=$ $\Phi\left(x_{1}\right)=x_{2}$ and similarly $\left[h, y_{2}\right]=-y_{2}$. The eigenspace for ad $h$ for the eigenvalues 1 and -1 are $k[h] x$ and $y k[h]$ respectively. Hence $x_{2}=b(h) x$ and $y_{2}=y c(h)$ for some $b, c \in k[h]$. Then

$$
x_{2} y_{2}=b(h) x y c(h)=b(h) a(h-1) c(h)
$$

and

$$
y_{2} x_{2}=y c(h) b(h) x=c(h+1) y x b(h+1)=c(h+1) a(h) b(h+1) .
$$

But

$$
x_{2} y_{2}=\Phi\left(x_{1} y_{1}\right)=\Phi\left(a_{1}(h-1)\right)=a_{1}(h+\tau-1)
$$

and

$$
y_{2} x_{2}=\Phi\left(y_{1} x_{1}\right)=\Phi\left(a_{1}(h)\right)=a_{1}(h+\tau) .
$$

Therefore $a_{1}(h+\tau)=c(h+1) a(h) b(h+1)$. Hence $\operatorname{deg} a_{1} \geq \operatorname{deg} a$ and, by symmetry, $\operatorname{deg} a \geq \operatorname{deg} a_{1}, A\left(a_{1}\right)$ being isomorphic to $A(a)$. Thus $\operatorname{deg} a_{1}=\operatorname{deg} a$ and $\operatorname{deg} c=$ $\operatorname{deg} b=0$. By monicity, $a_{1}(h+\tau)=a(h)$ and $b c=1$. Applying an automorphism from $\oint 3.5$ (i), we can assume that $c=b=1$, whence $\Phi\left(x_{1}\right)=x$ and $\Phi\left(y_{1}\right)=y$.

Now suppose that $\gamma=-1$, that is $\Phi\left(h_{1}\right)=\tau-h$. Here $\left[h, x_{2}\right]=-x_{2}$ and $\left[h, y_{2}\right]=y_{2}$ so $x_{2}=y b(h)$ and $y_{2}=c(h) x$ for some $b, c \in k[h]$. Then

$$
x_{2} y_{2}=y b(h) c(h) x=b(h+1) a(h) c(h+1)
$$

and

$$
y_{2} x_{2}=c(h) x y b(h)=c(h) a(h-1) b(h) .
$$

But $x_{2} y_{2}=a_{1}(\tau-h-1)$ and $y_{2} x_{2}=a_{1}(\tau-h)$. Therefore $a_{1}(\tau-h-1)=$ $b(h+1) a(h) c(h+1)$ and $\operatorname{deg} a_{1} \geq \operatorname{deg} a$. By symmetry, $\operatorname{deg} a_{1}=\operatorname{deg} a=d$ and $\operatorname{deg} c=\operatorname{deg} b=0$. By monicity, $a_{1}(\tau-h-1)=(-1)^{d} a(h)$ and $b c=(-1)^{d}$. We can assume that $b=1$ and $c=(-1)^{d}$, whence $\Phi\left(x_{1}\right)=y$ and $\Phi\left(y_{1}\right)=(-1)^{d} x$.

3.28. Theorem. For $a_{1}, a_{2} \in k[h], A\left(a_{1}\right) \simeq A\left(a_{2}\right)$ if and only if $a_{2}(h)=$ $\eta a_{1}(\tau \pm h)$ for some $\eta, \tau \in k$ with $\eta \neq 0$.

Proof. If $a_{1}$ and $a_{2}$ are monic, then this is immediate from Theorem 3.27. The general case reduces to the monic case on application of isomorphisms of the form given in Lemma 3.1(i).

3.29. Theorem. If $a$ is reflective, then $\operatorname{Aut}_{k} A(a)$ is generated by $G$ and $\Omega$. If $a$ is not reflective, then $\operatorname{Aut}_{k} A(a)=G$.

Proof. Let $\Gamma \in \mathrm{Aut}_{k} A(a)$. By Theorem[3.27, with $x_{1}=\Gamma(x), y_{1}=\Gamma(y), h_{1}=\Gamma(h)$ and $a_{1}=a$, there exist $\Phi \in G$ and $\tau \in k$ such that either $x=\Phi \Gamma(x), y=\Phi \Gamma(y)$, $h+\tau=\Phi \Gamma(h)$ and $a(h+\tau)=a(h)$ or $\Phi \Gamma(x)=y, \Phi \Gamma(y)=(-1)^{d} x, \Phi \Gamma(h)=\tau-h$ and $(-1)^{d} a(\tau-h-1)=a(h)$. In the latter case $a$ is reflective, with $\rho=\tau-1$, and $\Gamma=\Phi^{-1} \Omega \in\langle G, \Omega\rangle$. In the former case, which must hold if $a$ is not reflective, $\tau$ must be 0 and $\Gamma=\Phi^{-1} \in G$. 
3.30. Remark. When $d=2, \Omega \in G$; see [12, 4.4]. It would be interesting to know when $\Omega \in G$.

3.31. Proposition. Let $w \in A$. Then $w$ is strictly nilpotent if and only if there exists $\Phi \in G$ such that $\Phi(w) \in k[x]$ or $\Phi(w) \in k[y]$.

Proof. This result holds when $d \leq 2$ by [11, Théorème 9.1] and [12, Théorème 6.2], so we may assume that $d>2$. By Lemma 3.4, $x$ and $y$ are strictly nilpotent and it follows, as in [12, 4.1], that if there exists an automorphism $\Phi$ as stated, then $w$ is strictly nilpotent.

Suppose that $w$ is strictly nilpotent. By Lemma 3.25 there exists $\Phi \in G$ such that $\Phi(w) \in k[x]$ or $\Phi(w) \in k[y]$ or $\Phi(w)=\lambda x+\eta h+\mu y+\tau$ for some $\lambda, \eta, \mu, \tau \in k$. It suffices to show, in the third possibility, that $\eta=0$ and that $\lambda=0$ or $\mu=0$. Let $z=\Phi(w)$. In the $H_{1,1}$-grading, $\bar{z}=\lambda T^{d}+\mu Y^{d}$ so it follows from Lemma 3.20 that this is a monomial, that is $\lambda=0$ or $\mu=0$. If $\lambda=0$, then $y$ is an eigenvector for $\operatorname{ad} z$ with eigenvalue $-\eta$ and if $\mu=0$, then $x$ is an eigenvector for $\operatorname{ad} z$ with eigenvalue $\eta$. As $z$ is strictly nilpotent, $\eta=0$ as required.

3.32. Remark. When $a$ is reflective $\Omega(x)=y$ so the criterion for $w$ to be strictly nilpotent becomes the existence of $\Phi \in \operatorname{Aut}_{k} A$ such that $\Phi(w) \in k[x]$.

\section{Algebras similar to $U\left(\mathfrak{s l}_{2}\right)$}

Smith 31] considers a class of algebras similar to the enveloping algebra of $\mathfrak{s l}_{2}$. Let $f=f(H) \in k[H]$. We denote by $R(f)$ the $k$-algebra generated by $A, B$ and $H$ subject to the relations

$$
[H, A]=A,[H, B]=-B,[A, B]=f(H) .
$$

As an ambiskew polynomial ring, $R(f)=R(k[H], \sigma, f(H), 1)$, where $\sigma$ is the $k$-automorphism of $k[H]$ such that $\sigma(H)=H-1$. By Lemma $2.4 R(f)=$ $k[H, W](\sigma, W)$, where $\sigma(H)=H-1$ and $\sigma(W)=W+f(H)$.

We continue to assume that $\operatorname{char} k=0$. In [31] $k=\mathbb{C}$, but the proofs of those results we quote from [31 are valid more generally. By [31, Proposition 1.5], the centre of $R(f)$ is generated by the Casimir element

$$
C:=A B+B A+\frac{1}{2}(u(H+1)-u(H))=2 A B+u(H)=2 B A+u(H+1),
$$

where $u \in k[H]$ is such that $f(H)=u(H+1)-u(H)$ and is unique up to addition of scalars. Let $x, y$ and $h$ denote the images in $R(f) / C R(f)$ of $A, B$ and $H$ respectively. Then the $k$-algebra $R(f) / C R(f)$ is generated by $x, y, h$ subject to the relations

$$
x h=(h-1) x, y h=(h+1) y, x y=-\frac{1}{2} u(h), y x=-\frac{1}{2} u(h+1) .
$$

Thus $R(f) / C R(f)=A(a)$, where $a(h)=-\frac{1}{2} u(h+1)$. More generally, for $\gamma, \delta \in k$, with $\gamma \neq 0$,

$$
R(f) /(\gamma C-\delta) R(f)=A\left(\frac{\delta}{2 \gamma}-\frac{1}{2} u(h+1)\right) .
$$

In [31, Lemma 6.1 and Remark 2], Smith notes the first two parts of the following lemma and remarks that he believes it will be very difficult to understand precisely when $R\left(f_{1}\right) \simeq R\left(f_{2}\right)$.

4.1. Lemma. Let $f_{1}, f_{2} \in k[H]$.

(i) If $f_{2}=\gamma f_{1}$ for some non-zero $\gamma \in k$, then $R\left(f_{1}\right) \simeq R\left(f_{2}\right)$ via $A \mapsto \gamma^{-1} A, B \mapsto$ $B$ and $H \mapsto H$. 
(ii) If $f_{2}(H)=f_{1}(H+\tau)$ for some $\tau \in k$, then $R\left(f_{1}\right) \simeq R\left(f_{2}\right)$ via $A \mapsto A, B \mapsto B$ and $H \mapsto H+\tau$.

(iii) If $f_{2}(H)=f_{1}(-H)$, then $R\left(f_{1}\right) \simeq R\left(f_{2}\right)$ via $A \mapsto B, B \mapsto-A$, and $H \mapsto-H$.

Proof. (i), (ii) and (iii) are special cases of Lemma 2.8(i), (v) and (vi), respectively. In (iii), one applies 2.8 with $p=1$ and $\tau(H)=-H$.

Combining the three parts of Lemma 4.1, $R\left(f_{1}\right) \simeq R\left(f_{2}\right)$ if $f_{2}(H)=\gamma f_{1}(\tau \pm H)$ for some $\gamma, \tau \in k$ with $\gamma \neq 0$. We shall now show that this condition is also necessary for $R\left(f_{1}\right) \simeq R\left(f_{2}\right)$.

4.2. Theorem. For $f_{1}(H), f_{2}(H) \in k[H], R\left(f_{1}\right) \simeq R\left(f_{2}\right)$ if and only if $f_{2}(H)=$ $\eta f_{1}(\tau \pm H)$ for some $\eta, \tau \in k$ with $\eta \neq 0$.

Proof. Lemma 4.1 establishes that the given condition is sufficient for $R\left(f_{1}\right) \simeq$ $R\left(f_{2}\right)$. For necessity, suppose that there is an isomorphism $\theta: R\left(f_{1}\right) \rightarrow R\left(f_{2}\right)$. For $i=1,2$, let $C_{i}=2 A B+u_{i}(H)=2 B A+u_{i}(H+1) \in R\left(f_{i}\right)$, where $u_{i} \in k[H]$ is such that $f_{i}(H)=u_{i}(H+1)-u_{i}(H)$. The centre of $R_{i}$ is $k\left[C_{i}\right]$ and so there exist $\gamma, \delta \in k$, with $\gamma \neq 0$, such that $\theta\left(C_{1}\right)=\gamma C_{2}-\delta$. There is an induced isomorphism $R\left(f_{1}\right) / C_{1} R\left(f_{1}\right) \simeq R\left(f_{2}\right) /\left(\gamma C_{2}-\delta\right) R\left(f_{2}\right)$ so, by (30),

$$
A\left(-\frac{1}{2} v_{1}\right) \simeq A\left(\sigma, \frac{\delta}{2 \gamma}-\frac{1}{2} v_{2}\right)
$$

where each $v_{i}(h)=u_{i}(h+1)$. By Lemma 3.1 (i),

$$
A\left(v_{1}\right) \simeq A\left(\rho+v_{2}\right)
$$

where $\rho=-\frac{\delta}{\gamma} \in k$. By Theorem 3.28 , there exist $\eta, \tau \in k$, with $\eta \neq 0$, such that $\rho+v_{2}(h)=\eta v_{1}(\tau \pm h)$. Then

$$
\begin{aligned}
f_{2}(H) & =u_{2}(H+1)-u_{2}(H) \\
& =v_{2}(H)-v_{2}(H-1) \\
& =\left(v_{2}(H)+\rho\right)-\left(v_{2}(H-1)+\rho\right) \\
& =\eta v_{1}(\tau \pm H)-\eta v_{1}(\tau \pm(H-1)) \\
& =\eta\left(u_{1}(\tau \pm H+1)-u_{1}(\tau \pm H \mp 1+1)\right) \\
& =\eta f_{1}(\tau+H) \text { or }-\eta f_{1}(1+\tau-H) .
\end{aligned}
$$

\section{Algebras similar to the Primitive factors of $U_{q}\left(\mathfrak{s l}_{2}\right)$}

In this section we concentrate on the case where $D$ is the Laurent polynomial ring $k\left[h^{ \pm 1}\right]$ and $\sigma(h)=q h$, where $q \in k^{*}$ is not a root of unity. Let $a=a(h) \in k\left[h^{ \pm 1}\right]$ and let $W=k\left[h^{ \pm 1}\right](\sigma, a)$. Thus $W$ is the $k$-algebra generated by $h, h^{-1}, x$ and $y$ subject to the relations

$$
x h=q h x, y h=q^{-1} h y, x y=a(q h), y x=a(h) .
$$

Examples include the minimal primitive factors of the quantized enveloping algebra $U_{q}\left(\mathfrak{s l}_{2}\right)$, for which the details will be given in Example 5.3. These algebras may be viewed as quantizations of those considered in Section 3 but the isomorphism problem is influenced by the existence of the unit $h$. The following lemma lists some routine consequences of the definitions, Lemma [2.7(ii) and the fact that $k\left[h^{ \pm 1}\right]$ is a domain. Here $U(W)$ denotes the group of units of $W$. 
5.1. Lemma. (i) If $a \in k\left[h^{ \pm 1}\right]$ is a non-zero non-unit, then $U(W) / k^{*}$ is cyclic, generated by $h$.

(ii) If $a=\lambda h^{n}$ is a unit in $k\left[h^{ \pm 1}\right]$, then $U(W) / k^{*}$ is free abelian of rank two, generated by $h$ and $x$, and $W$ is isomorphic to the skew Laurent polynomial ring $k\left[h^{ \pm 1}\right]\left[x^{ \pm 1} ; \sigma\right]$.

(iii) For each positive integer $n,\left\{w \in W: h^{-1} w h=q^{n} w\right\}=k\left[h^{ \pm 1}\right] x^{n}$ and $\left\{w \in W: h^{-1} w h=q^{-n} w\right\}=y^{n} k\left[h^{ \pm 1}\right]$.

5.2. Theorem. Let $0 \neq a_{1}, a_{2} \in k\left[h^{ \pm 1}\right]$, let $q \in k^{*}$ and let $\sigma \in \operatorname{Aut}_{k} k\left[h^{ \pm 1}\right]$ be such that $\sigma(h)=q h$. Then $k\left[h^{ \pm 1}\right]\left(\sigma, a_{1}\right) \simeq k\left[h^{ \pm 1}\right]\left(\sigma, a_{2}\right)$ if and only if $a_{2}(h)=$ $\eta h^{m} a_{1}\left(\mu h^{ \pm 1}\right)$ for some $\eta, \mu \in k^{*}$ and some integer $m$.

Proof. For $i=1,2$, let $W_{i}=k\left[h^{ \pm 1}\right]\left(\sigma, a_{i}\right)$. By Lemma 5.1, we can assume that $a_{1}$ and $a_{2}$ are non-units; if both are units, then the stated condition holds and, by [5.1 (ii), $W_{1} \simeq k\left[h^{ \pm 1}\right]\left[x^{ \pm 1} ; \sigma\right] \simeq W_{2}$, while if only one is a unit, then the stated condition fails and, by $\left[5.1\right.$ (i) and (ii), $W_{1} \nsucceq W_{2}$.

If $a_{2}(h)=\eta h^{m} a_{1}(\mu h)$, then, by Lemma2.7(ii), with $\lambda=\eta h^{m}$, and Lemma 2.7(v) with $\tau(h)=\mu h, W_{1} \simeq W_{2}$ with

$$
x \mapsto x \eta^{-1} h^{-m}, y \mapsto y \text { and } h \mapsto \mu h .
$$

If $a_{2}(h)=\eta h^{m} a_{1}\left(\mu h^{-1}\right)$, then, by Lemma 2.7(ii) and Lemma 2.7(vi), with $\tau(h)=$ $q^{-1} \mu h^{-1}$ so that $\tau \sigma(h)=\mu h^{-1}=\sigma^{-1} \tau(h), W_{1} \simeq W_{2}$ with

$$
x \mapsto y, y \mapsto x \eta^{-1} h^{-m} \text {, and } h \mapsto q^{-1} \mu h^{-1} .
$$

For the converse, suppose that there is an isomorphism $\Gamma: W_{1} \rightarrow W_{2}$. By Lemma 5.1(i), there exists $\tau \in k^{*}$ such that $\Gamma(h)=\tau h^{ \pm 1}$. Suppose first that $\Gamma(h)=\tau h$. By Lemma 5.1(iii), there exist $f_{1}, g_{1} \in k\left[h^{ \pm 1}\right]$ such that $\Gamma(x)=f_{1}(h) x$ and $\Gamma(y)=y g_{1}(h)$. In $W_{2}$,

$$
\begin{aligned}
a_{1}(\tau h) & =\Gamma\left(a_{1}(h)\right)=\Gamma(y x)=y g_{1}(h) f_{1}(h) x \\
& =g_{1}\left(q^{-1} h\right) f_{1}\left(q^{-1} h\right) y x=g_{1}\left(q^{-1} h\right) f_{1}\left(q^{-1} h\right) a_{2}(h) .
\end{aligned}
$$

Hence

$$
a_{1}(h)=g_{1}\left(\tau^{-1} q^{-1} h\right) f_{1}\left(\tau^{-1} q^{-1} h\right) a_{2}\left(\tau^{-1} h\right) .
$$

By symmetry, there exist $f_{2}, g_{2} \in k\left[h^{ \pm 1}\right]$ such that

$$
a_{2}(h)=g_{2}\left(\tau q^{-1} h\right) f_{2}\left(\tau q^{-1} h\right) a_{1}(\tau h) .
$$

Using (32) to substitute in (31) for $a_{2}\left(\tau^{-1} h\right)$, we obtain

$$
a_{1}(h)=g_{1}\left(\tau^{-1} q^{-1} h\right) f_{1}\left(\tau^{-1} q^{-1} h\right) g_{2}\left(q^{-1} h\right) f_{2}\left(q^{-1} h\right) a_{1}(h) .
$$

From this it follows that $f_{1}, f_{2}, g_{1}$ and $g_{2}$ are all units in $k\left[h^{ \pm 1}\right]$ and hence, by (32), that $a_{2}(h)=\eta h^{m} a_{1}(\tau h)$ for some $\eta \in k^{*}$ and some integer $m$.

Now suppose that $\Gamma(h)=\tau h^{-1}$. By Lemma [5.1(iii), there exist $f_{1}, g_{1} \in k\left[h^{ \pm 1}\right]$ such that $\Gamma(y)=f_{1}(h) x$ and $\Gamma(x)=y g_{1}(h)$. In $W_{2}$,

$$
\begin{aligned}
a_{1}\left(\tau h^{-1}\right) & =\Gamma\left(a_{1}(h)\right)=\Gamma(y x) \\
& =f_{1}(h) x_{y} g_{1}(h)=f_{1}(h) a_{2}\left(q h^{-1}\right) g_{1}(h) .
\end{aligned}
$$

Hence

$$
a_{1}(h)=f_{1}\left(\tau^{-1} h^{-1}\right) g_{1}\left(\tau^{-1} h^{-1}\right) a_{2}\left(q \tau^{-1} h^{-1}\right)
$$


By symmetry, there exist $f_{2}, g_{2} \in k\left[h^{ \pm 1}\right]$ such that

$$
a_{2}(h)=f_{2}\left(\tau^{-1} h^{-1}\right) g_{2}\left(\tau^{-1} h^{-1}\right) a_{1}\left(q \tau^{-1} h^{-1}\right) .
$$

Using (32) to substitute in (31) for $a_{2}\left(q \tau^{-1} h^{-1}\right)$, we obtain

$$
a_{1}(h)=f_{1}\left(\tau^{-1} h^{-1}\right) g_{1}\left(\tau^{-1} h^{-1}\right) f_{2}\left(q^{-1} h\right) g_{2}\left(q^{-1} h\right) a_{1}(h) .
$$

It follows that $f_{1}, f_{2}, g_{1}$ and $g_{2}$ are all units in $k\left[h^{ \pm 1}\right]$ and hence, by (34), that $a_{2}(h)=\eta h^{m} a_{1}\left(q \tau^{-1} h^{-1}\right)$ for some $\eta \in k^{*}$ and some integer $m$.

5.3. Example. When $q$ is not a root of unity, the minimal primitive ideals of the quantized enveloping algebra $U_{q}\left(\mathfrak{s l}_{2}\right)$ are generalized Weyl algebras of the form $C_{\mu}:=k\left[t^{ \pm 1}\right](\sigma, u-\mu)$, where $\sigma(t)=q^{2} t, \mu \in k$ and $u=-\frac{q^{-2} t^{2}+q^{2} t^{-2}}{\left(q^{2}-q^{-2}\right)^{2}}$; see [16, 7.9]. A simple calculation based on Theorem 5.2 shows that, for $\lambda, \mu \in k, C_{\lambda} \simeq C_{\mu}$ if and only if $\mu= \pm \lambda$. There are isomorphisms $\Gamma_{1}, \Gamma_{2}: C_{\lambda} \rightarrow C_{-\lambda}$ such that

$$
\Gamma_{1}(x)=-x, \Gamma_{1}(y)=y \text { and } \Gamma_{1}(t)=i t
$$

and

$$
\Gamma_{2}(x)=-y, \Gamma_{2}(y)=x \text { and } \Gamma_{2}(t)=i q^{2} t^{-1} .
$$

The first of these was noted in [16, 7.9] for two particular values of $\lambda$, namely those for which $C_{\lambda}$ has infinite global dimension.

Because of the term $h^{m}$, Theorem 5.2 cannot be lifted in the way that Theorem 3.28 was lifted modulo a central element to yield Theorem 4.2 In the analogue of Theorem 4.2, the term $h^{m}$ disappears.

5.4. Theorem. For $i=1,2$, let $R_{i}$ be the $k$-algebra generated by $H^{ \pm 1}, X$ and $Y$ subject to the relations

$$
X H=q H X, Y H=q^{-1} H Y, X Y-Y X=v_{i}(H),
$$

where $q \in k^{*}$ is not a root of unity and $0 \neq v_{i}(H) \in k\left[H^{ \pm 1}\right]$. Suppose that both $v_{1}$ and $v_{2}$ have zero constant term. Then $R_{1} \simeq R_{2}$ if and only if $v_{2}(H)=\eta v_{1}\left(\mu H^{ \pm 1}\right)$ for some $\eta, \mu \in k^{*}$.

Proof. Each $R_{i}$ is an ambiskew polynomial ring, $R\left(k\left[H^{ \pm 1}\right], \sigma, v_{i}, 1\right)$, where $\sigma(H)=$ $q H$. By the condition on constant terms, there exist $u_{1}, u_{2} \in k\left[H^{ \pm 1}\right]$ such that each $v_{i}=u_{i}(q H)-u_{i}(H)$. For $i=1,2$, let $Z_{i}=Y X-u_{i}(H)=X Y-u_{i}(q H)$. By [17, 2.1(ii)], for $i=1,2, k\left[Z_{i}\right]$ is the centre of $R_{i}$. As a generalized Weyl algebra, $R_{i}=k\left[H^{ \pm 1}, Z\right]\left(\sigma, Z+u_{i}\right)$, where $\sigma(H)=q H$ and $\sigma(Z)=Z$.

Suppose that there is a $k$-isomorphism $\Gamma: R_{1} \rightarrow R_{2}$. It is easy to see that the units of $R_{i}$ have the form $\lambda H^{m}$, where $\lambda \in k^{*}$ and $m \in \mathbb{Z}$. Hence there exists $\lambda \in k^{*}$ such that $\Gamma(H)=\lambda H^{ \pm 1}$.

There exist $\beta \in k^{*}$ and $\gamma \in k$ such that $\Gamma\left(Z_{1}\right)=\beta Z_{2}+\gamma$. Then

$$
\Gamma(Y X)=\Gamma\left(Z_{1}+u_{1}(H)\right)=\beta Z_{2}+\gamma+u_{1}\left(\lambda H^{ \pm 1}\right) .
$$

It follows from consideration of degrees in $Y$ and $X$ in the iterated skew polynomial ring $R_{2}=k\left[H^{ \pm 1}\right][X ; \sigma]\left[Y ; \sigma^{-1}, \delta\right]$ that one of $\Gamma(X)$ and $\Gamma(Y)$ has the form $f_{1}(H) Y+g_{1}(H), f_{1}, g_{1} \in k\left[H^{ \pm 1}\right]$, and the other has the form $X f_{2}(H)+g_{2}(H)$, $f_{2}, g_{2} \in k\left[H^{ \pm 1}\right]$. 
Suppose that $\Gamma(H)=\lambda H$. As $H^{-1} X H=q X$ and $H^{-1} Y H=q^{-1} Y$, we must have $\Gamma(X)=X f_{2}(H)$ and $\Gamma(Y)=f_{1}(H) Y$. By (35),

$$
f_{1}(H) f_{2}(H) Y X=\Gamma(Y X)=\beta\left(Y X-u_{2}(H)\right)+\gamma+u_{1}(\lambda H),
$$

whence $u_{2}(H)=\beta^{-1}\left(u_{1}(\lambda H)+\gamma\right)$ and $v_{2}(H)=\beta^{-1} v_{1}(\lambda H)$.

Now suppose that $\Gamma(H)=\lambda H^{-1}$. In this case we must have $\Gamma(X)=f_{1}(H) Y$ and $\Gamma(Y)=X f_{2}(H)$. By (35),

$$
f_{2}(q H) f_{1}(q H) X Y=p^{-1} \beta\left(X Y-u_{2}(q H)\right)+\gamma+u_{1}\left(\lambda H^{-1}\right),
$$

whence $u_{2}(H)=p \beta^{-1}\left(u_{1}\left(\lambda H^{-1}\right)+\gamma\right)$ and $v_{2}(H)=-p \beta^{-1} v_{1}\left(\lambda q^{-1} H^{-1}\right)$. In both cases, $v_{2}(H)=\eta v_{1}\left(\mu H^{ \pm 1}\right)$ for some $\eta, \mu \in k^{*}$.

For the converse, if $v_{2}(H)=\eta v_{1}(\mu H)$ for some $\eta, \mu \in k^{*}$, then $R_{1} \simeq R_{2}$ by Lemma 2.8(i) with $\lambda=\eta$ and Lemma 2.8(v) with $\tau(H)=\mu H$. If $v_{2}(H)=$ $\eta v_{1}\left(\mu H^{-1}\right)$ for some $\eta, \mu \in k^{*}$, then $R_{1} \simeq R_{2}$ by Lemma 2.8(i) with $\lambda=\eta$ and Lemma 2.8(vi) with $\tau(H)=\mu H^{-1}$.

\section{The DEFormations of Witten And Woronowicz}

6.1. Notation. In this section we consider a class of algebras including two algebras named in [10, 35] as Woronowicz's deformation and Witten's second deformation. We offer two proofs that these two algebras are isomorphic. One is based on an analysis of this class of algebras and the second is a direct application of Lemma 2.7 based on the identification of Woronowicz's deformation and Witten's second deformation as generalized Weyl algebras in [8].

We consider ambiskew polynomial rings of the form $R(k[t], \sigma, v, p)$, where, for some $q \in k^{*}$ and $c \in k, \sigma$ is the $k$-automorphism of $k[t]$ such that $\sigma(t)=q t+c$ and $v=d t+e \in k[t]$ has degree 1 . Such a ring $A$ is the $k$-algebra generated by $x, y$ and $t$ subject to the relations

$$
x t-q t x=c x, y t-q^{-1} t y=-q^{-1} c y, x y-p y x=d t+e .
$$

By Lemma 2.4 $A$ is the generalized Weyl algebra $k[t, w](\sigma, w)$, where $\sigma$ is extended to the polynomial ring $k[t, w]$ by setting $\sigma(w)=p w+v$. If $p \neq q$ and either $e=0$ or $p \neq 1$, then there exists $u=f t+g \in k[t]$ of degree 1 such that $v=\sigma(u)-p u$, whence, by Lemma 2.5)(ii), $A=k[t, z](\sigma, f t+z+g)$, where $\sigma(z)=p z$.

6.2. Example. Taking $q=r^{2}, c=-r, d=1, e=0$ and $p=s^{2}$, where $r, s \in k^{*}$, one obtains a class of algebras discussed by Fairlie [13. The defining relations are

$$
r t x-r^{-1} x t=x, r y t-r^{-1} t y=y, s^{-1} x y-s y x=t .
$$

In [13, the generators $x, y, t$ are written as $W_{+}, W_{-}$and $W_{0}$ respectively. We shall denote the algebra with these generators and relations by $F_{s, r}$. For Woronowicz's deformation, introduced in [34, p. 150] and discussed in the survey article [30], take $r=\nu^{2}$ and $s=\nu \in k^{*}$. In [34, 30], $k=\mathbb{C}$ and $\nu \in[-1,1]$ is real. The generators $\nabla_{0}, \nabla_{1}$ and $\nabla_{2}$ given in [34, 30] are related to ours by the formulae

$$
\nabla_{1}=\left(1+\nu^{2}\right) t, \nabla_{0}=\left(1+\nu^{2}\right) x, \nabla_{2}=-y .
$$

For Witten's second deformation, introduced in [33, 5.13], take $r=q^{\frac{1}{2}}$ and $s=q \in$ $k^{*}$. In 33], the generators $x, y, t$ are written as $t_{+}, t_{-}$and $t_{0}$ respectively. 
6.3. Notation. For a given pair of parameters $p$ and $q$ with $q \neq 1$, the algebras specified in $\$ 6.1$ fall into two isomorphism classes and there is a simple procedure, outlined in the proof of the next theorem, to determine which isomorphism class a given algebra is in. Let $\sigma$ be the $k$-automorphism of $k[t]$ such that $\sigma(t)=q t$. Let $W_{p, q}=R(k[t], \sigma, t+1, p)$ and $V_{p, q}=R(k[t], \sigma, t, p)$. If $p \neq q$, then by Lemma 2.5(ii), $V_{p, q}=k[t, z](\sigma, z+t /(q-p))$ and, if also $p \neq 1, W_{p, q}=k[t, z](\sigma, z+t /(q-p)+1 /(1-$ $p)$ ), where $\sigma(z)=p z$. By Lemma 2.4. $W_{1, q}=k[t, w](\sigma, w)$, where $\sigma(w)=w+t+1$.

6.4. Theorem. Let $A$ be one of the rings specified in 6.1 . If $q \neq 1$, then either $A \simeq W_{p, q}$ or $A \simeq V_{p, q}$.

Proof. Let $t^{\prime}=t+\frac{c}{q-1}$. Then $\sigma\left(t^{\prime}\right)=q t^{\prime}$ and $A=R\left(k\left[t^{\prime}\right], \sigma, d t^{\prime}+e^{\prime}, p\right)$ where $e^{\prime}=e-\frac{d c}{q-1}$.

If $e^{\prime}=0$, then there is an isomorphism from $A$ to $V_{p, q}$ given by $t^{\prime} \mapsto d^{-1} t, x \mapsto x$ and $y \mapsto y$.

If $e^{\prime} \neq 0$, then there is an isomorphism from $A$ to $W_{p, q}$ given by $t^{\prime} \mapsto e^{\prime} d^{-1} t, x \mapsto$ $e^{\prime} x$ and $y \mapsto y$.

The next lemma indicates symmetry in the roles of the pairs $(t, q)$ and $(z, p)$ in $W_{p, q}$ and $V_{p, q}$.

6.5. Lemma. (i) $V_{q, p} \simeq V_{p, q}$ and, if $q \neq 1$ and $p \neq 1, W_{q, p} \simeq W_{p, q}$.

(ii) $W_{p, q} \simeq W_{p^{-1}, q^{-1}}$ and $V_{p, q} \simeq V_{p^{-1}, q^{-1}}$.

Proof. (i) We may assume that $p \neq q$. We have observed that $V_{p, q}=k[t, z](\sigma, z+$ $t /(q-p))$ and, if $p \neq 1, W_{p, q}=k[t, z](\sigma, z+t /(q-p)+1 /(1-p))$, where $\sigma(t)=q t$ and $\sigma(z)=p z$. Applying Lemma [2.7)(iv) with $\tau(z)=t /(q-p)$ and $\tau(t)=(q-p) z$, so that $\tau \sigma \tau^{-1}(z)=q z, \tau \sigma \tau^{-1}(t)=p t$ and $\tau(z+t /(q-p))=z+t /(q-p)$, we obtain $V_{p, q} \simeq V_{q, p}$ and, if $p \neq 1$ and $q \neq 1, W_{p, q} \simeq W_{q, p}$.

(ii) follows easily from Lemma 2.8(i).

6.6. Remark. In 6.1, if $q=1$, then $A \simeq R(k[t], \sigma, t, p)$, where $\sigma=$ id or $\sigma(t)=t-1$, depending on whether $c=0$. In particular, $W_{p, 1} \simeq V_{p, 1}$ which, by Lemma 6.5(i), is isomorphic to $V_{1, p}$. However, if $p \neq 1, W_{1, p}$ and $V_{1, p}$ are not isomorphic. It can be deduced from [22, 3.1] that, if $p \neq 1, W_{1, p}$ has a unique 1-dimensional simple module whereas $V_{1, p}$ has infinitely many, because in this case $k[t, z]$ has a maximal ideal invariant under $\sigma$.

6.7. Example. We apply the algorithm from the proof of Theorem 6.4 to the algebras $F_{s, r}$ in Example 6.2 assuming that $r^{2} \neq 1$. In the notation of that proof, $t^{\prime}=t-\frac{r}{r^{2}-1}$ and $e^{\prime}=\frac{r}{r^{2}-1} \neq 0$. Consequently, $F_{s, r} \simeq W_{s^{2}, r^{2}}$.

6.8. Corollary. Let $q=\nu^{2} \in k^{*}$. If $q^{2} \neq 1$, then Woronowicz's deformation and Witten's second deformation are isomorphic algebras.

Proof. From the discussions in Examples 6.2 and 6.7 Woronowicz's deformation $F_{\nu, \nu^{2}}$ is isomorphic to $W_{\nu^{2}, \nu^{4}}$ while Witten's second deformation $F_{q, q^{1 / 2}}$ is isomorphic to $W_{q^{2}, q}=W_{\nu^{4}, \nu^{2}}$. By Lemma 6.5(i), the two are isomorphic.

An alternative proof is based upon the identifications of the two deformations as generalized Weyl algebras given by the first author [8]. Let $D$ be the commutative polynomial algebra $k[C, H]$ and let $\sigma$ be the $k$-automorphism of $D$ such that $\sigma(C)=$ $r^{4} C$ and $\sigma(H)=r^{2} H$. From [8], Woronowicz's deformation is $D(\sigma, a)$, where $a=$ $C-\left(H-r /\left(1-r^{2}\right)\right)\left(H-r^{3} /\left(1-r^{2}\right)\right) / r^{2}\left(r+r^{-1}\right)$, and Witten's second deformation 
is $D\left(\sigma, a_{1}\right)$, where $a_{1}=H+\mu C+\rho$ for some $\mu, \rho \in k^{*}$. By straightforward changes of generators, we can assume that $a=C-\lambda H^{2}+H+1$ for some $\lambda \in k^{*}$ and that $a_{1}=C+H+1$.

Let $\tau$ be the $k$-automorphism of $D$ such that $\tau(C)=C-\lambda H^{2}$ and $\tau(H)=H$. Then $\sigma \tau=\tau \sigma$ and $\tau(a)=a_{1}$ so, by Lemma 2.7(v), $D(\sigma, a) \simeq D\left(\sigma, a_{1}\right)$.

6.9. Remark. The algebra known as Witten's first deformation [10, 35], which was introduced in [33. 5.2], is of the form $R(k[t], \sigma, v, p)=k[t, w](\sigma, w)$, with $p=1$, specified in 6.1 except that $v$ is quadratic in $t$. The algebraic relationship between Witten's two deformations was explained in rigorous terms by Le Bruyn [24]. Below we apply his method more generally, using a change of variables of the form used in the proof of Theorem 6.4 and observe that the drop in degree from 2 to 1 in the degree of the element $v$ in the passage from Witten's first deformation to his second is, in a sense, singular.

Let $A$ be a $k$-algebra of the form $R(k[t], \sigma, v, p)$, where $p, q \in k^{*}, q \neq 1, \sigma(t)=$ $q t+c$ and $v \in k[t]$ has degree 2. As in the proof of Theorem [6.4, a change of variables allows us to assume that $c=0$. Thus $A$ is the $k$-algebra generated by $x, y$ and $t$ subject to the relations

$$
x t=q t x, y t=q^{-1} t y, x y-p y x=f t^{2}+d t+e,
$$

for some $f, d, e \in k$ with $f \neq 0$. The homogenization $B$, say, of $A$ is the graded $k$-algebra generated by $x, y, t$ and $z$ subject to the relations

$$
\begin{gathered}
x t=q t x, y t=q^{-1} t y, x y-p y x=f t^{2}+d t z+e z^{2}, \\
z x=x z, z y=y z, z t=t z .
\end{gathered}
$$

The element $t$ is normal in $B$ and the automorphism $\tau$ of $B$ for which $t b=\tau(b) t$ for all $b \in B$ is given by

$$
x \mapsto q^{-1} x, y \mapsto q y, t \mapsto t, z \mapsto z .
$$

Following [2, $\S 8$ ], the twist $B_{\tau}$ of $B$ by $\tau$ is the graded $k$-algebra which is isomorphic to $B$ as a graded abelian group, with an isomorphism written as $a \mapsto a_{\tau}$, and has multiplication determined by the rule $a_{\tau} b_{\tau}=\left(\tau^{d}(a) b\right)_{\tau}$, where $a, b \in B$ are homogeneous and $d=\operatorname{deg}(b)$. Thus $B_{\tau}$ is the $k$-algebra generated by $x_{\tau}, y_{\tau}, t_{\tau}$ and $z_{\tau}$ subject to the relations

$$
\begin{gathered}
x_{\tau} t_{\tau}=t_{\tau} x_{\tau}, y_{\tau} t_{\tau}=t_{\tau} y_{\tau}, x_{\tau} y_{\tau}-p^{2} q^{-2} y_{\tau} x_{\tau}=q^{-1}\left(f t_{\tau}^{2}+d t_{\tau} z_{\tau}+e z_{\tau}^{2}\right), \\
z_{\tau} x_{\tau}=q x_{\tau} z_{\tau}, z_{\tau} y_{\tau}=q^{-1} y_{\tau} z_{\tau}, z_{\tau} t_{\tau}=t_{\tau} z_{\tau} .
\end{gathered}
$$

The element $t_{\tau}$ is central and the dehomogenization $C$, say, of $B_{\tau}$ is obtained by factoring out the ideal generated by $t_{\tau}-1$. Thus it is the $k$-algebra generated by $x_{\tau}, y_{\tau}$ and $z_{\tau}$ subject to the relations

$$
z_{\tau} x_{\tau}=q x_{\tau} z_{\tau}, z_{\tau} y_{\tau}=q^{-1} y_{\tau} z_{\tau}, x_{\tau} y_{\tau}-p^{2} q^{-2} y_{\tau} x_{\tau}=q^{-1}\left(f+d z_{\tau}+e z_{\tau}^{2}\right) .
$$

Comparing these with (36), the defining parameters $q$ and $p$ are replaced by $q^{-1}$ and $p^{2} q^{-2}$ respectively. If $e \neq 0$, the algebra $C$ is of the same general form as $A$ with $v$ quadratic, but if $e=0$, it is of the original form specified in $\$ 6.1$ with the degree of $v$ dropping from 2 to 1 . This is the situation with Witten's two deformations. His first deformation is of the form $A$, with $v$ quadratic with zero constant term, both before and after the change of variables which makes $t$ normal, and his second is the corresponding algebra formed by homogenization, twisting and dehomogenization. 
The original parameters, labelled $q$ and $p$ in the general discussion above, are $q^{-1}$ and 1 and so become $q$ and $q^{2}$ in $C$.

We conclude this section by showing that, under certain conditions on the parameters, the only situations where there are isomorphisms between algebras of the forms $V_{p, q}$ and $W_{p, q}$ are those covered by Remark 6.6 and Lemma 6.5

6.10. Theorem. Let $p, q, p_{1}, q_{1} \in k^{*} \backslash\{1\}$ be such that the subgroups $\langle p, q\rangle$ and $\left\langle p_{1}, q_{1}\right\rangle$ of $k^{*}$ have positive rank, $p \neq q$ and $p_{1} \neq q_{1}$.

(i) $W_{p, q} \nsucceq V_{p_{1}, q_{1}}$.

(ii) If $\left\{p_{1}, q_{1}\right\} \neq\{p, q\}$ and $\left\{p_{1}, q_{1}\right\} \neq\left\{p^{-1}, q^{-1}\right\}$, then $W_{p, q} \neq W_{p_{1}, q_{1}}$ and $V_{p, q} \neq$ $V_{p_{1}, q_{1}}$.

Proof. (i) This follows from the determination of the finite-dimensional simple modules for $V_{p, q}$ and $W_{p, q}$ given by [22, 3.1]. In the notation of [22], $v_{d}=\left(1+p q^{-1}+\right.$ $\left.\ldots+\left(p q^{-1}\right)^{d-1}\right) t$ for $V_{p, q}$ and $v_{d}=\left(1+p q^{-1}+\ldots+\left(p q^{-1}\right)^{d-1}\right) t+1+p+\ldots+p^{d-1}$ for $W_{p, q}$. The only positive integers $s$ for which there exist $s$-dimensional simple $V_{p, q}$-modules are 1 , for which there are infinitely many, and, if it exists, the least positive integer $d$ such that $q^{d}=p^{d}$, for which there are again infinitely many. On the other hand, $W_{p, q}$ also has infinitely many 1 -dimensional simple $W_{p, q}$-modules but, for $d>1$, there is at most one $d$-dimensional simple module. For $d=2$, this module exists precisely when $\left(\left(1+p q^{-1}\right) t+1+p\right) k[t] \neq(t+1) k[t]$. As $q \neq 1$, there is a unique two-dimensional simple $W_{p, q}$-module.

(ii) We begin with the determination of the non-zero normal elements which generate prime ideals of $W_{p, q}$. Let $W=W_{p, q}$ and let $S=R\left(k\left[t^{ \pm 1}\right], \sigma, t, p\right)$ be the localization of $W$ at the powers of the normal element $t$. As $p \neq 1$ and $p \neq q$, the element $z$ of Lemma 2.5 exists and the results of [17] apply. Both $z W$ and $t W$ are prime ideals of $W$. Let $\mathcal{N}(W)=\{w \in W: w W=W w$ is a non-zero prime ideal of $W$ \} and define $\mathcal{N}(S)$ in a similar way.

First consider the case where the subgroup $\langle p, q\rangle$ of $k^{*}$ has rank two. By [17. 2.21], $\mathcal{N}(S)$ consists of associates of $z$ and hence, by standard localization theory, for example [26, 2.1.16], $\mathcal{N}(W)=k^{*} z \cup k^{*} t$.

Now consider the case where $\langle p, q\rangle$ has rank one. Without loss of generality, we may assume that $q$ is not a root of unity so that [17, 2.21] is applicable to $S$. Let $n$ be the minimal positive integer such that $p^{n} \in\langle q\rangle$ and let $i$ be the integer such that $p^{n}=q^{i}$. If $i<0$, then $t^{-i} z^{n}$ is central in $W$ and, for all $\eta \in k^{*}, t^{-i} z^{n}-\eta \in \mathcal{N}(W)$. If $i \geq 0$, then $t^{i}-\eta z^{n} \in \mathcal{N}(W)$ for all $\eta \in k^{*}$. It follows from [17] 2.21] that $\mathcal{N}(S)$ consists of the associates of $z$ and those of elements of the form $t^{-i} z^{n}-\eta$ or $t^{i}-\eta z^{n}, \eta \in k^{*}$, depending on whether $i>0$. It then follows that $\mathcal{N}(W)=$ $k^{*} z \cup k^{*} t \cup\left(\bigcup_{\eta \in k^{*}} k^{*}\left(t^{-i} z^{n}-\eta\right)\right)$ if $i<0$ and $\mathcal{N}(W)=k^{*} z \cup k^{*} t \cup\left(\bigcup_{\eta \in k^{*}} k^{*}\left(t^{i}-\eta z^{n}\right)\right)$ if $i>0$.

In either case, for $m \geq 0$, let $W_{m}=k[t, z] x^{m}$ and $W_{-m}=y^{m} k[t, z]$. Then $W=$ $\sum_{m \in \mathbb{Z}} W_{m}$ and each $W_{m}$ is simultaneously an eigenspace for all the automorphisms of $W$ of the form $w \mapsto a^{-1} w a, a \in \mathcal{N}(W)$. For each $j \in \mathbb{Z}$, the eigenvalues for $W_{j}$ are $q^{j}$ for $a=t, p^{j}$ for $a=z, 1$ when $a$ has the form $t^{-i} z^{n}-\eta$ and $p^{j n}=q^{j i}$ when $a$ has the form $t^{i}-\eta z^{n}$. It follows that the set $\left\{\{p, q\},\left\{p^{-1}, q^{-1}\right\}\right\}$ is determined by $W$. This proves the result for $W_{p, q}$ and the proof for $V_{p, q}$ is similar.

\section{REFERENCES}

[1] S. A. Amitsur, Commutative linear differential operators, Pacific J. Math. 8 (1958), 1-10. MR 20:1808 
[2] M. Artin, J. Tate and M. Van den Bergh, Modules over regular algebras of dimension 3, Invent. Math. 106 (1991), 335-388. MR 93e:16055

[3] V. V. Bavula, Finite-dimensionality of Ext ${ }^{n}$ and Tor $_{n}$ of simple modules over a class of algebras, Funktsional. Anal. i Prilozhen. 25 (1991), no. 3, 80-82. MR 92k:16019

[4] V. V. Bavula, Generalized Weyl algebras and their representations, Algebra i Analiz 4 (1992), no. 1, 75-97; English transl. in St Petersburg Math. J. 4 (1993), 71-92. MR 93h:16043

[5] V. V. Bavula, Description of two-sided ideals in a class of noncommutative rings, I, Ukrainian Math. J. 45 (1993), no. 2, 223-234. MR 94h:16053

[6] V. V. Bavula, Description of two-sided ideals in a class of noncommutative rings, II, Ukrainian Math. J. 45 (1993), no. 3, 329-334. MR 94h:16054

[7] V. V. Bavula, Generalized Weyl algebras, kernel and tensor-simple algebras, their simple modules, CMS Conf. Proc. 14 (1993), 83-107. CMP 94:09

[8] V. V. Bavula, Global dimension of Generalized Weyl algebras, CMS Conf. Proc. 18 (1996), 81-107. MR 97e:16018

[9] V. V. Bavula, Tensor homological minimal algebras, global dimension of the tensor product of algebras and of generalized Weyl algebras, Bull. Sci. Math., 120 (1996), no. 3, 293-335. MR 97f:16015

[10] T. L. Curtright and C. Zachos, Deforming maps for quantum algebras, Phys. Lett. B 243, no. 3, (1990), 237-244. MR 91h:17020

[11] J. Dixmier, Sur les algèbres de Weyl, Bull. Soc. Math. France 96 (1968), 209-242. MR 39:4224

[12] J. Dixmier, Quotients simples de l'algèbre enveloppante de $\mathfrak{s l}_{2}$, J. Algebra 24 (1973), 551-564. MR 46:9134

[13] D. Fairlie, Quantum deformations of SU(2), J. Phys. A A23 (1990) L183-L187. MR 91f:17011

[14] T. J. Hodges, Non-commutative deformations of type-A Kleinian singularities, J. Algebra 161 (1993), 271-290. MR 94i:14038

[15] D. A. Jordan, Iterated skew polynomial rings and quantum groups, J. Algebra 156 (1993), 194-218. MR 94b:16034

[16] D. A. Jordan Krull and global dimension of certain iterated skew polynomial rings, Abelian Groups and Noncommutative Rings, a Collection of Papers in Memory of Robert B. Warfield, Jr., Contemp. Math. 130 (1992), 201-213. MR 93h:16052

[17] D. A. Jordan, Height one prime ideals of certain iterated skew polynomial rings, Math. Proc. Cambridge Philos. Soc. 114 (1993), 407-425. MR 94i:16013

[18] D. A. Jordan, Primitivity in skew Laurent polynomial rings and related rings, Math. Z. 213 (1993), 353-371. MR 94k:16009

[19] D. A. Jordan, Finite-dimensional simple modules over certain iterated skew polynomial rings, J. Pure Appl. Algebra 98 (1995), 45-55. MR 96g:16037

[20] D. A. Jordan, A simple localization of the quantized Weyl algebra, J. Algebra 174 (1995), 267-281. MR 96m:16035

[21] D. A. Jordan, Down-up algebras and ambiskew polynomial rings, J. Algebra 228 (2000), 311-346.

[22] D. A. Jordan and I. E. Wells, Invariants for automorphisms of certain iterated skew polynomial rings, Proc. Edinburgh Math. Soc. 39 (1996), 461-472. MR 97k:16038

[23] G. R. Krause and T.H. Lenagan, Growth of algebras and Gelfand-Kirillov dimension, Pitman, London (1985). MR 86g:16001

[24] L. Le Bruyn, Two remarks on Witten's quantum enveloping algebra, Comm. Algebra 22 no.3 (1994), 865-876. MR 94m:17015

[25] J. C. McConnell and J. C. Robson, Homomorphisms and extensions of modules over certain differential polynomial rings, J. Algebra 26 (1973), 319-342. MR 49:7312

[26] J. C. McConnell and J. C. Robson, Noncommutative Noetherian rings, Wiley, Chichester (1987). MR 89j:16023

[27] C. Năstăsescu and F. Van Oystaeyen, Graded Ring Theory, North-Holland, Amsterdam (1982). MR 84i:16002

[28] A. L. Rosenberg, Noncommutative algebraic geometry and representations of quantized algebras, Kluwer, Dordrecht (1995). MR 97b:14004

[29] L. Rowen, Ring Theory, Vol. I, Academic Press, San Diego (1988). MR 89h:16001 
[30] S. P. Smith, Quantum groups. An introduction and survey for ring theorists, Noncommutative rings, MSRI Pub. 24 (1992), Springer, Berlin, Heidelberg, New York, 131-178. MR 94g:17032

[31] S. P. Smith, A class of algebras similar to the enveloping algebra of sl(2), Trans. Amer. Math. Soc. 322 (1990), 285-314. MR 91b:17013

[32] J. T. Stafford, Homological properties of the enveloping algebra of $s l(2)$, Math. Proc. Cambridge Philos. Soc. 91 (1982), 29-37. MR 83h:17013

[33] E. Witten, Gauge theories, vertex models, and quantum groups, Nuclear Phys. B B330 (1990), 285-346. MR 92b:82051

[34] S. L. Woronowicz, Twisted SU(2)-group. An example of a non-commutative differential calculus, Publ. Res. Inst. Math. Sci., 23 (1987), 117-181. MR 88h:46130

[35] C. Zachos, Elementary paradigms of quantum algebras, Deformation theory and quantum groups with applications to Mathematical Physics, Contemp. Math. 134 (1992), 351-377. MR 94k:81137

Department of Mathematics and Computer Science, University of Antwerp, U. I. A., B-2610 WilriJk, Belgium

Current address: Department of Pure Mathematics, University of Sheffield, Hicks Building, Sheffield S3 7RH, United Kingdom

E-mail address: v.bavula@sheffield.ac.uk

Department of Pure Mathematics, University of Sheffield, Hicks Building, Sheffield S3 7RH, United KINGDOM

E-mail address: d.a.jordan@sheffield.ac.uk 